\title{
Passivity analysis and passive control for uncertain discrete switched time-delay systems via a simple switching signal design
}

\author{
Ker-Wei Yu ${ }^{1,2}$, Chang-Hua Lien ${ }^{1 *}$, Jenq-Der Chen ${ }^{3}$ and Long-Yeu Chung ${ }^{4}$
}

"Correspondence:

chlien@mail.nkmu.edu.tw

1 Department of Marine

Engineering, National Kaohsiung Marine University, Kaohsiung, 811, Taiwan, ROC

Full list of author information is available at the end of the article

\begin{abstract}
In this paper, we consider the problem for passivity analysis and passive control of uncertain discrete switched systems with interval time-varying delay and linear fractional perturbations via a simple switching signal design. A new Lyapunov-Krasovskii functional is used to propose some LMI conditions that design the switching signal to guarantee the passivity and passive switching control of discrete switched time-delay systems. Jensen and Park inequalities combined with delay-partitioning approach are investigated to improve the conservativeness of the obtained results. Finally, some numerical examples and a water quality model illustrate the main proposed results.
\end{abstract}

Keywords: switching signal; passivity analysis; passive switching control; internal stability; discrete switched systems; interval time-varying delay; delay-partitioning approach

\section{Introduction}

The linear control systems and the complex or uncertain feedback systems can be bridged by the framework of switched linear systems [1]. The switched system is an important class of hybrid systems, which consists of some subsystems and a switching signal. The switching signal will handle the switching among subsystems. Many complicate nonlinear system behaviors can be produced under switching, such as multiple limit cycles and chaos $[1,2]$. Switched systems are often encountered in many practical systems including automated highway systems, automotive engine control systems, chemical process, constrained robotics, mutlirate control, power systems and power electronics, robot manufacture, stepper motors, and water quality control [1-4]. It is also well known that instability or bad performance may be introduced by the existence of delay in a system [5-7]. Time-delay phenomena are often confronted in many practical engineering systems, such as chemical engineering systems, hydraulic systems, inferred grinding models, neural network circuits, nuclear reactor, and rolling mill systems. Hence, the problems of stability and stabilization for discrete switched systems with time delay have been investigated in recent years $[1,2,8-17]$.

In the recent years, there are two interesting and well-known issues investigated for switched systems. (1) The stability property for each subsystem cannot guarantee that 
the overall system is stable under arbitrary switching $[1,2,10,14-16]$. (2) The stability of a switched system may be achieved by selecting the switching signal even when each subsystem is unstable $[1,2,11,13,18-23]$. In [13], a design of switching signal is proposed to ensure the stability and stabilization of discrete switched systems with interval time-varying delay. In [16], the switching is identified to guarantee the stability of discrete switched time-delay system. In recent years, the passivity property was provided in diverse aspects, such as complexity analysis [24], fuzzy control [25], time-delay systems [1, 26-29], neural networks systems [29, 30], and signal processing [31]. The passivity theory was first introduced in circuit analysis, which is a promising approach to keep the internal stability of systems. Furthermore, linear fractional perturbations in [11, 18, 19, 32, 33] are more generalized than parameter ones in $[10,14,15,29,30]$. In this paper, a simple design scheme for switching signal in passivity analysis and passive control is proposed for discrete switched systems with interval time-varying delay and linear fractional perturbations. In [34], a delay-partitioning approach was proposed to improve the conservativeness of the developed results. In this paper, a new delay-partitioning approach is investigated to make more accurate evaluation for the allowable upper bound of interval time-varying delay. In [35], Jensen and Park inequalities are used to improve the main proposed results. To the best knowledge of the authors, results that combine the delaypartitioning approach and Jensen and Park inequalities are not reported in the past. Some numerical examples are made to demonstrate the obtained results. The practical application of a water quality model is also provided to illustrate the proposed results. From the simulation result, our proposed approach in this paper provides less conservative results. The main contribution of this paper can be highlighted as follows:

(1) The less conservative passivity analysis and passive switching control for discrete switched systems with linear fractional perturbations and interval time-varying delay via a switching signal design are considered.

(2) The proposed approach provides less LMI variables and a shorter program running time than some previous ones in the past.

(3) Jensen and Park inequalities combined with the delay-partitioning approach are used to improve the conservativeness of the developed results.

(4) The proposed design scheme for switching signal is more flexible than those in [12-14]. The proposed approach for switching signal design scheme can be easily applied to continuous switched time-delay systems.

In the past, some new relevant results and approaches had been proposed to achieve the performance of switched systems [36-39]. In [36], the Borne-Gentina practical stability of continuous switched systems is considered by the aggregation techniques. In [37], the stability and $H_{\infty}$ control of switched systems with interval time-varying delay are guaranteed by input-output scaled small gain theorem approach. In [38], the stability for discrete switched nonlinear systems with unstable subsystems is considered by the T-S fuzzy model approach. In [39], the passivity of discrete switched nonlinear systems is studied by multiple storage functions and multiple supply rates approach. The proposed results in this paper can be considered and improved by the above developed approaches.

The notation used throughout this paper is as follows. For a matrix $A$, we denote the transpose by $A^{T}$, symmetric positive (negative) definite by $A>0(A<0) ; A \leq B$ $(A<B)$ means that $B-A$ is a symmetric positive semidefinite (definite) matrix; $I$ de- 
notes the identity matrix. Define $\bar{N}=\{1,2, \ldots, N\}, A \backslash B=\{x \mid x \in A$ and $x \notin B\}, L_{2}(0, \infty)=$ $\left\{w(k) \mid \sum_{k=0}^{\infty} w^{T}(k) w(k)<\infty\right\}$.

\section{Problem statement and preliminaries}

In this paper, we consider the following uncertain discrete switched system with time delay:

$$
\begin{aligned}
& x(k+1)= {\left[A_{\sigma}+\Delta A_{\sigma}(k)\right] x(k)+\left[B_{\sigma}+\Delta B_{\sigma}(k)\right] x(k-r(k)) } \\
&+\left[D_{\sigma}+\Delta D_{\sigma}(k)\right] w(k), \\
& z(k)= {\left[A_{z \sigma}+\Delta A_{z \sigma}(k)\right] x(k)+\left[B_{z \sigma}+\Delta B_{z \sigma}(k)\right] x(k-r(k)) } \\
&+ {\left[D_{z \sigma}+\Delta D_{z \sigma}(k)\right] w(k), } \\
& x(\theta)=\varphi(\theta), \quad \theta=-r_{M},-r_{M}+1, \ldots, 0,
\end{aligned}
$$

where $x(k) \in \Re^{n}, x_{k}$ is the state defined by $x_{k}(\theta):=x(k+\theta), \forall \theta \in\left\{-r_{M},-r_{M}+1, \ldots, 0\right\}$, $w(k) \in \mathfrak{R}^{p}$ is a disturbance input, $z(k) \in \mathfrak{R}^{p}$ is a regulated output, $\sigma$ is a switching signal in the finite set $\{1,2, \ldots, N\}$ and will be selected to preserve the performance of the system, $\varphi(k) \in \Re^{n}$ denotes the initial function, the time-varying delay $r(k)$ is a function from $\{0,1,2,3, \ldots\}$ to $\{1,2,3, \ldots\}$ such that $0<r_{m} \leq r(k) \leq r_{M}$, where $r_{m}$ and $r_{M}$ are two given positive integers. The matrices $A_{i}, B_{i}, D_{i}, A_{z i}, B_{z i}, D_{z i}, i=1,2, \ldots, N$, are given constant matrices of appropriate dimensions; $\Delta A_{i}(k), \Delta B_{i}(k), \Delta D_{i}(k), \Delta A_{z i}(k), \Delta B_{z i}(k)$, and $\Delta D_{z i}(k)$ are some perturbed matrices satisfying the following conditions:

$$
\begin{aligned}
& {\left[\begin{array}{lll}
\Delta A_{i}(k) & \Delta B_{i}(k) & \Delta D_{i}(k)
\end{array}\right]=M_{i} \cdot \Delta_{i}(k) \cdot\left[\begin{array}{lll}
N_{A i} & N_{B i} & N_{D i}
\end{array}\right],} \\
& {\left[\begin{array}{lll}
\Delta A_{z i}(k) & \Delta B_{z i}(k) & \Delta D_{z i}(k)
\end{array}\right]=M_{z i} \cdot \Delta_{z i}(k) \cdot\left[\begin{array}{lll}
N_{Z A i} & N_{Z B i} & N_{Z D i}
\end{array}\right],} \\
& \Delta_{i}(k)=\left[\begin{array}{ll}
I-\Gamma_{i}(k) \Xi_{i}
\end{array}\right]^{-1} \Gamma_{i}(k), \quad \Xi_{i} \Xi_{i}^{T}<I, \\
& \Delta_{z i}(k)=\left[\begin{array}{ll}
I-\Gamma_{z i}(k) \Xi_{z i}
\end{array}\right]^{-1} \Gamma_{z i}(k), \quad \Xi_{z i} \Xi_{z i}^{T}<I,
\end{aligned}
$$

where $M_{i} \in \Re^{n \times q}, M_{z i} \in \Re^{p \times q}, N_{A i}, N_{B i}, N_{D i}, N_{Z A i}, N_{Z B i}$, and $N_{Z D i}, i=1,2, \ldots, N, \Xi_{i}$ and $\Xi_{z i}$ are some given constant matrices of appropriate dimensions; and $\Gamma_{i}(k)$ and $\Gamma_{z i}(k)$ are some unknown matrices satisfying

$$
\Gamma_{i}^{T}(k) \Gamma_{i}(k) \leq I, \quad \Gamma_{z i}^{T}(k) \Gamma_{z i}(k) \leq I
$$

Now we propose the following switching domains:

$$
\Omega_{i}\left(U_{i}\right)=\left\{x \in \Re^{n}: x^{T} U_{i} x \geq 0\right\}, \quad i=1,2, \ldots, N
$$

where the matrices $U_{i}>0, i=1,2, \ldots, N$, will be selected from our developed results, and

$$
\begin{aligned}
& \bar{\Omega}_{1}=\Omega_{1}, \quad \bar{\Omega}_{2}=\Omega_{2} \backslash \bar{\Omega}_{1}, \quad \bar{\Omega}_{3}=\Omega_{3} \backslash \bar{\Omega}_{1} \backslash \bar{\Omega}_{2}, \quad \ldots \\
& \bar{\Omega}_{N}=\Omega_{N} \backslash \bar{\Omega}_{1} \backslash \ldots \backslash \bar{\Omega}_{N-1} .
\end{aligned}
$$


From the above definition of the domains, the switching signal can be selected by

$$
\sigma(x(k))=i, \quad \forall x(k) \in \bar{\Omega}_{i}
$$

where $\bar{\Omega}_{i}$ is defined in (2b).

The following lemmas will be used to obtain the main proposed result.

Lemma 1 If there exist some constants $0 \leq \alpha_{i} \leq 1, i \in \bar{N}, \sum_{i=1}^{N} \alpha_{i}=1$, some matrices $U_{i}>0$, $i \in \bar{N}$, such that

$$
\sum_{i=1}^{N} \alpha_{i} \cdot U_{i}>0
$$

we have

$$
\bigcup_{i=1}^{N} \bar{\Omega}_{i}=\Re^{n} \quad \text { and } \quad \bar{\Omega}_{i} \cap \bar{\Omega}_{j}=\Phi, \quad \forall i \neq j,
$$

where $\Phi$ is the empty set, and $\bar{\Omega}_{i}$ is defined in $(2 \mathrm{~b})$.

Proof By the definition of $\bar{\Omega}_{i}$ in (2b), $\bar{\Omega}_{i} \cap \bar{\Omega}_{j}=\Phi$ is trivial. For any $x \in \Re^{n}$ with $\sum_{i=1}^{N} \alpha_{i} \cdot U_{i}>$ 0 , we have

$$
x^{T}\left[\sum_{i=1}^{N} \alpha_{i} \cdot U_{i}\right] x=\sum_{i=1}^{N} \alpha_{i} \cdot x^{T} U_{i} x \geq 0, \quad \forall x \in \Re^{n} .
$$

This condition implies

$$
x^{T} U_{i} x \geq 0 \quad \text { for some } i \in \bar{N} \text {. }
$$

From $(2 a)$ and $(2 b)$ we have

$$
\begin{array}{ll}
x \in \Omega_{i}\left(U_{i}\right) & \text { for some } i \in \bar{N} \\
x \in \bar{\Omega}_{j}\left(U_{j}\right) & \text { for some } i, j \in \bar{N}, i \geq j .
\end{array}
$$

The proof is completed.

Remark 1 In the recent years, some proposed switching domains are shown as follows:

(a) In [13], the switching domains are selected as

$$
\Omega_{i}\left(P, U, A_{i}\right)=\left\{x \in \Re^{n}: x^{T}\left[\left(r_{M}-r_{m}\right) \cdot U-A_{i}^{T} P-P A_{i}\right] x<0\right\}, \quad i=1,2, \ldots, N
$$

where the matrices $P>0, U>0, \bar{\Omega}_{1}=\Omega_{1}, \bar{\Omega}_{2}=\Omega_{2} \backslash \bar{\Omega}_{1}, \ldots, \bar{\Omega}_{N}=\Omega_{N} \backslash\left(\bigcup_{i=1}^{N-1} \bar{\Omega}_{i}\right)$.

(b) In [18], the switching domains are selected as

$$
\Omega_{i}\left(P, U, A_{i}\right)=\left\{x \in \Re^{n}: x^{T}\left(A_{i}^{T} P A_{i}\right) x \leq x^{T} U x\right\}, \quad i=1,2, \ldots, N,
$$

where the matrices $P>0, U>0, \bar{\Omega}_{1}=\Omega_{1}, \bar{\Omega}_{2}=\Omega_{2} \backslash \bar{\Omega}_{1}, \ldots, \bar{\Omega}_{N}=\Omega_{N} \backslash \bar{\Omega}_{1} \backslash \cdots \backslash \bar{\Omega}_{N-1}$. 
(c) In [19], the switching domains are selected as

$$
\Omega_{i}\left(P, U, A_{i}\right)=\left\{x \in \Re^{n}: x^{T}\left(A_{i}^{T} P A_{i}\right) x \leq x^{T} U_{i} x\right\}, \quad i=1,2, \ldots, N
$$

where the matrices $P>0, U_{i}>0, i=1,2, \ldots, N$, and $\bar{\Omega}_{i}$ is defined in (b).

In this paper, the switching domains are defined in (2a)-(2b) with

$$
\Omega_{i}\left(U_{i}\right)=\left\{x \in \Re^{n}: x^{T} U_{i} x \geq 0\right\},
$$

where the matrices $U_{i}=U_{i}^{T}, i=1,2, \ldots, N$. The proposed approach in this paper does not depend on the system parameters $A_{i}$, which shows that the proposed scheme is more simple and flexible than the above results. It can be easily extended to continuous switched systems to design the switching signal under consideration.

Lemma 2 [40] For a given symmetric matrix $S=\left[\begin{array}{cc}S_{11} & S_{12} \\ * & S_{22}\end{array}\right]$, the following conditions are equivalent:

(1) $S<0$,

(2) $S_{22}<0, S_{11}-S_{12} S_{22}^{-1} S_{12}^{T}<0$.

Lemma 3 [32] Suppose that $\Delta_{i}(k)$ is defined in (1f) and satisfies (1h). Then for real matrices $U_{i}, W_{i}$, and $X_{i}$ with $X_{i}=X_{i}^{T}$, the following conditions are equivalent:

(I) The following inequality is satisfied:

$$
X_{i}+U_{i} \Delta_{i}(k) W_{i}+W_{i}^{T} \Delta_{i}^{T}(k) U_{i}^{T}<0
$$

(II) There exists a scalar $\varepsilon_{i}>0$ such that

$$
\left[\begin{array}{ccc}
X_{i} & U_{i} & \varepsilon_{i} \cdot W_{i}^{T} \\
* & -\varepsilon_{i} \cdot I & \varepsilon_{i} \cdot \Xi_{i}^{T} \\
* & * & -\varepsilon_{i} \cdot I
\end{array}\right]<0
$$

where the matrix $\Xi_{i}$ is defined in (1f).

Lemma 4 (Discrete Jensen inequality [35]) For any matrix $R>0$, integers $r_{1}<r_{2}$, and a vector function $\omega(i) \in \Re^{n}$, the following inequality is satisfied:

$$
-\left(r_{2}-r_{1}\right) \cdot \sum_{i=k-r_{2}}^{k-r_{1}-1} \omega^{T}(i) R \omega(i) \leq-\left[\sum_{i=k-r_{2}}^{k-r_{1}-1} \omega(i)\right]^{T} R\left[\sum_{i=k-r_{2}}^{k-r_{1}-1} \omega(i)\right] .
$$

Lemma 5 (Park inequality in [35]) For any matrices $V \in \Re^{n \times n}>0, M_{1}, M_{2} \in \Re^{n \times m}$, a positive real number $0<\alpha<1$, and a vector $\omega \in \Re^{m}$, there exists a matrix $X \in \Re^{n \times n}$ such that

$$
\left[\begin{array}{ll}
V & X \\
* & V
\end{array}\right]>0
$$


Then the following inequality is satisfied:

$$
-\left[\frac{1}{\alpha} \omega^{T} M_{1}^{T} V M_{1} \omega+\frac{1}{1-\alpha} \omega^{T} M_{2}^{T} V M_{2} \omega\right] \leq-\omega^{T}\left[\begin{array}{l}
M_{1} \\
M_{2}
\end{array}\right]^{T}\left[\begin{array}{cc}
V & X \\
* & V
\end{array}\right]\left[\begin{array}{l}
M_{1} \\
M_{2}
\end{array}\right] \omega .
$$

Definition 1 The discrete switched system of (1a)-(1h) with the switching signal in (2c) is called passive if there exists a constant $\gamma>0$ such that

$$
-\gamma \cdot \sum_{k=0}^{\ell} w^{T}(k) w(k) \leq 2 \cdot \sum_{k=0}^{\ell} z^{T}(k) w(k)
$$

for all $\ell>0$ and zero initial condition $x(\theta)=0, \theta=-r_{M},-r_{M}+1, \ldots, 0$. If the parameter $\ell$ is selected as $\infty$, then the disturbance input $w$ belongs to $L_{2}(0, \infty)$.

In this paper, the following partitions are selected:

$$
0<r_{m}=r_{0}<r_{1}<r_{2}<\cdots<r_{p-1}<r_{p}=r_{M},
$$

where $p$ is the partition number, and $r_{i}, i=1,2, \ldots, p$, are some positive integers.

Theorem 1 For some selected integers $0<r_{m}=r_{0}<r_{1}<r_{2}<\cdots<r_{p-1}<r_{p}=r_{M}$, constants $0 \leq \alpha_{j} \leq 1, j=1,2, \ldots, N$, and $\sum_{i=1}^{N} \alpha_{i}=1$, system (1a)-(1h) is passive by the designed switching signal in (2c) if there exist some $n \times n$ symmetric matrices $P>0, Q_{1}>0, Q_{2}>0, R_{1}>0$, $R_{2}>0, R_{3}>0, S>0, T>0, V_{1}>0, V_{2}>0, U_{j}, W_{1}, W_{2}$, an $n \times n$ matrix $X$, and constants $\varepsilon_{j}>0, j=1,2, \ldots, N$ such that the following LMI conditions are satisfied:

$$
\begin{aligned}
& R_{2}+W_{1}>0, \quad R_{2}+W_{2}>0, \\
& {\left[\begin{array}{cc}
Q_{1} & W_{1} \\
* & Q_{2}
\end{array}\right]>0, \quad\left[\begin{array}{cc}
Q_{1} & W_{2} \\
* & Q_{2}
\end{array}\right]>0, \quad\left[\begin{array}{cc}
V_{1} & X \\
* & V_{1}
\end{array}\right]>0,} \\
& \Sigma_{j}^{i}=\left[\begin{array}{cc}
\Sigma_{1 j}^{i} & \Sigma_{2 j} \\
* & \Sigma_{3 j}
\end{array}\right]<0, \quad i=1,2, \ldots, p, j=1,2, \ldots, N, \\
& \sum_{i=1}^{N} \alpha_{i} \cdot U_{i}>0,
\end{aligned}
$$

where $\Sigma_{1 j}^{i}, \Sigma_{2 j}, \Sigma_{3 j}, \Sigma_{11 j}, \Sigma_{13 j}, \Sigma_{14 j}$ are defined by

$$
\begin{aligned}
\Sigma_{1 j}^{i}= & {\left[\begin{array}{ccccc}
\Sigma_{11 j} & 0 & \Sigma_{13 j} & \Sigma_{14 j} & \Sigma_{15 j} \\
* & \Sigma_{22 j}^{i} & \Sigma_{23 j}^{i} & \Sigma_{24 j}^{i} & \Sigma_{25 j} \\
* & * & \Sigma_{33 j}^{i} & \Sigma_{34 j} & 0 \\
* & * & * & \Sigma_{44 j}^{i} & 0 \\
* & * & * & * & \Sigma_{55 j}
\end{array}\right], } \\
\Sigma_{2 j} & =\left[\begin{array}{cccccc}
\Sigma_{16 j} & \Sigma_{17 j} & 0 & 0 & \Sigma_{110 j} & \Sigma_{111 j} \\
\Sigma_{26 j} & \Sigma_{27 j} & 0 & 0 & \Sigma_{210 j} & \Sigma_{211 j} \\
0 & 0 & 0 & 0 & 0 & 0 \\
0 & 0 & 0 & 0 & 0 & 0 \\
\Sigma_{56 j} & \Sigma_{57 j} & 0 & \Sigma_{59 j} & \Sigma_{510 j} & \Sigma_{511 j}
\end{array}\right],
\end{aligned}
$$




$$
\begin{aligned}
& \Sigma_{3 j}=\left[\begin{array}{cccccc}
\Sigma_{66 j} & 0 & \Sigma_{68 j} & 0 & 0 & 0 \\
* & \Sigma_{77 j} & \Sigma_{78 j} & 0 & 0 & 0 \\
* & * & \Sigma_{88 j} & 0 & \Sigma_{810 j} & 0 \\
* & * & * & \Sigma_{99 j} & 0 & \Sigma_{911 j} \\
* & * & * & * & \Sigma_{1010 j} & 0 \\
* & * & * & * & * & \Sigma_{1111 j}
\end{array}\right] \\
& i=1,2, \ldots, p, j=1,2, \ldots, N \\
& \Sigma_{11 j}=-P+T+\left(r_{M}-r_{m}\right)^{2} \cdot Q_{1}-R_{1}-R_{3}+U_{j} \\
& \Sigma_{13 j}=R_{1}, \quad \Sigma_{14 j}=R_{3}, \quad \Sigma_{15 j}=-A_{z j}^{T} \text {, } \\
& \Theta=r_{M}^{2} \cdot R_{1}+\left(r_{M}-r_{m}\right)^{2}\left(Q_{2}+R_{2}+V_{1}+V_{2}\right)+r_{m}^{2} \cdot R_{3}, \\
& \Sigma_{16 j}=\left(A_{j}-I\right)^{T} \Theta, \quad \Sigma_{17 j}=A_{j}^{T} P, \quad \Sigma_{110}=\varepsilon_{j} \cdot N_{A j}^{T} \text {, } \\
& \Sigma_{111 j}=\varepsilon_{j} \cdot N_{Z A j}^{T}, \quad \gamma_{23}^{i}=\frac{\left(r_{M}-r_{m}\right)}{\left(r_{M}-r_{i-1}\right)}, \quad \gamma_{24}^{i}=\frac{\left(r_{M}-r_{m}\right)}{\left(r_{i}-r_{m}\right)}, \\
& \Sigma_{22 j}^{i}=-\gamma_{23}^{i} \cdot\left(R_{2}+W_{2}\right)-\gamma_{24}^{i} \cdot\left(R_{2}+W_{1}\right)-\left(r_{M}-r_{m}\right) \cdot\left(W_{1}-W_{2}\right)-2 V_{1}+X+X^{T} \text {, } \\
& \Sigma_{23 j}^{i}=\gamma_{23}^{i} \cdot\left(R_{2}+W_{2}\right)+V_{1}-X^{T}, \\
& \Sigma_{24}^{i}=\gamma_{24}^{i} \cdot\left(R_{2}+W_{1}\right)-X+V_{1}, \quad \Sigma_{25 j}=-B_{z j}^{T}, \\
& \Sigma_{26 j}=B_{j}^{T} \Theta, \quad \Sigma_{27 j}=B_{j}^{T} P, \quad \Sigma_{210 j}=\varepsilon_{j} \cdot N_{B j}^{T}, \quad \Sigma_{211 j}=\varepsilon_{1 j} \cdot N_{Z B j}^{T} \text {, } \\
& \Sigma_{33 j}^{i}=-S-R_{1}-\gamma_{23}^{i} \cdot\left(R_{2}+W_{2}\right)-\left(r_{M}-r_{m}\right) \cdot W_{2}-V_{1}-V_{2}, \quad \Sigma_{34 j}=V_{2}+X, \\
& \Sigma_{44 j}^{i}=-(T-S)-\gamma_{24}^{i} \cdot\left(R_{2}+W_{1}\right)-R_{3}+\left(r_{M}-r_{m}\right) \cdot W_{1}-V_{1}-V_{2}, \\
& \Sigma_{55 j}=-D_{z j}-D_{z j}^{T}-\gamma \cdot I, \quad \Sigma_{56 j}=D_{j}^{T} \Theta, \quad \Sigma_{57 j}=D_{j}^{T} P, \quad \Sigma_{59 j}=-M_{z j}, \\
& \Sigma_{510 j}=\varepsilon_{j} \cdot N_{D j}^{T}, \quad \Pi_{511 j}=\varepsilon_{2 j} \cdot N_{Z D j}^{T}, \quad \Sigma_{66 j}=-\Theta, \quad \Sigma_{68 j}=\Theta^{T} M_{j}, \\
& \Sigma_{77 j}=-P, \quad \Sigma_{78 j}=P M_{j}, \quad \Sigma_{88 j}=-\varepsilon_{j} \cdot I, \quad \Sigma_{810 j}=\varepsilon_{j} \cdot \Xi_{j}^{T}, \\
& \Sigma_{99 j}=-\varepsilon_{j} \cdot I, \quad \Sigma_{911 j}=\varepsilon_{1 j} \cdot \Xi_{z j}^{T}, \quad \Sigma_{1010 j}=-\varepsilon_{j} \cdot I, \quad \Sigma_{1111 j}=-\varepsilon_{j} \cdot I .
\end{aligned}
$$

Proof Define the Lyapunov-Krasovskii functional by

$$
\begin{aligned}
V\left(x_{k}\right)= & x^{T}(k) P x(k)+\left(r_{M}-r_{m}\right) \cdot \sum_{j=-r_{M}+1}^{-r_{m}} \sum_{i=k-1+j}^{k-1} z^{T}(i) \hat{Q} z(i) \\
& +r_{M} \cdot \sum_{j=-r_{M}+1}^{0} \sum_{i=k-1+j}^{k-1} y^{T}(i) R_{1} y(i) \\
& +\left(r_{M}-r_{m}\right) \cdot \sum_{j=-r_{M}+1}^{-r_{m}} \sum_{i=k-1+j}^{k-1} y^{T}(i)\left[R_{2}+V_{1}+V_{2}\right] y(i) \\
& +r_{m} \cdot \sum_{j=-r_{m}+1}^{0} \sum_{i=k-1+j}^{k-1} y^{T}(i) R_{3} y(i)+\sum_{i=k-r_{M}}^{k-1-r_{m}} x^{T}(i) S x(i)+\sum_{i=k-r_{m}}^{k-1} x^{T}(i) T x(i),
\end{aligned}
$$

where $P>0, \hat{Q}=\operatorname{diag}\left[Q_{1} Q_{2}\right]>0, R_{1}>0, R_{2}>0, R_{3}>0, V_{1}>0, S>0, T>0, y(i)=x(i+1)-$ $x(i)$, and $z(i)=\left[x(i)^{T} y(i)^{T}\right]^{T}$. The difference of functional (4) along the solutions of system 
(1a)-(1h) has the form

$$
\begin{aligned}
\Delta V\left(x_{k}\right)= & V\left(x_{k+1}\right)-V\left(x_{k}\right) \\
= & \left.x^{T}(k+1) P x(k+1)-x^{T}(k) P x(k)\right]+\left(r_{M}-r_{m}\right)^{2} \cdot z^{T}(k) \hat{Q} z(k) \\
& -\left(r_{M}-r_{m}\right) \cdot \sum_{i=k-r_{M}}^{k-r_{m}-1} z^{T}(i) \hat{Q} z(i)+r_{M}^{2} \cdot y^{T}(k) R_{1} y(k) \\
& -r_{M} \cdot \sum_{i=k-r_{M}}^{k-1} y^{T}(i) R_{1} y(i)+\left(r_{M}-r_{m}\right)^{2} \cdot y^{T}(k)\left[R_{2}+V_{1}+V_{2}\right] y(k) \\
& -\left(r_{M}-r_{m}\right) \cdot \sum_{i=k-r_{M}}^{k-r_{m}-1} y^{T}(i)\left[R_{2}+V_{1}+V_{2}\right] y(i)+r_{m}^{2} \cdot y^{T}(k) R_{3} y(k) \\
& -r_{m} \cdot \sum_{i=k-r_{m}}^{k-1} y^{T}(i) R_{3} y(i)+x^{T}\left(k-r_{m}\right) S x\left(k-r_{m}\right) \\
& -x^{T}\left(k-r_{M}\right) S x\left(k-r_{M}\right)+\left[x^{T}(k) T x(k)-x^{T}\left(k-r_{m}\right) T x\left(k-r_{m}\right)\right] .
\end{aligned}
$$

By the definitions $y(i)=x(i+1)-x(i)$ and $z(i)=\left[x(i)^{T} y(i)^{T}\right]^{T}$ we have

$$
\begin{gathered}
-\sum_{i=k-r_{M}}^{k-r_{m}-1} z^{T}(i) \hat{Q} z(i)=-\sum_{i=k-r_{M}}^{k-r(k)-1}\left[\begin{array}{l}
x(i) \\
y(i)
\end{array}\right]^{T}\left[\begin{array}{ll}
Q_{1} & 0 \\
0 & Q_{2}
\end{array}\right]\left[\begin{array}{l}
x(i) \\
y(i)
\end{array}\right] \\
-\sum_{i=k-r(k)}^{k-r_{m}-1}\left[\begin{array}{l}
x(i) \\
y(i)
\end{array}\right]^{T}\left[\begin{array}{ll}
Q_{1} & 0 \\
0 & Q_{2}
\end{array}\right]\left[\begin{array}{l}
x(i) \\
y(i)
\end{array}\right], \\
\lambda_{1}=\left[x^{T}\left(k-r_{m}\right) W_{1} x\left(k-r_{m}\right)-x^{T}(k-r(k)) W_{1} x(k-r(k))\right] \\
-\sum_{i=k-r(k)}^{k-r_{m}-1}\left[y^{T}(i) W_{1} y(i)+2 x^{T}(i) W_{1} y(i)\right]=0, \\
\lambda_{2}=\left[x^{T}(k-r(k)) W_{2} x(k-r(k))-x^{T}\left(k-r_{M}\right) W_{2} x\left(k-r_{M}\right)\right] \\
-\sum_{k-r(k)-1}^{k}\left[y^{T}(i) W_{2} y(i)+2 x^{T}(i) W_{2} y(i)\right]=0 .
\end{gathered}
$$

From the previous derivations we obtain the following result:

$$
\begin{aligned}
\Delta V\left(x_{k}\right)+\left(r_{M}-r_{m}\right) \cdot\left(\lambda_{1}+\lambda_{2}\right)+\left[-2 z^{T}(k) w(k)-\gamma \cdot w^{T}(k) w(k)\right] \\
=x^{T}(k+1) P x(k+1)-x^{T}(k)[P-T] x(k)+\left(r_{M}-r_{m}\right)^{2} \cdot x^{T}(k) Q_{1} x(k) \\
\quad-\left(r_{M}-r_{m}\right) \cdot x^{T}(k-r(k))\left[W_{1}-W_{2}\right] x(k-r(k)) \\
\quad+[x(k+1)-x(k)]^{T}\left[r_{M}^{2} \cdot R_{1}+\left(r_{M}-r_{m}\right)^{2}\left(Q_{2}+R_{2}+V_{1}+V\right)+r_{m}^{2} \cdot R_{3}\right] \\
\quad \cdot[x(k+1)-x(k)]-r_{M} \cdot \sum_{i=k-r_{M}}^{k-1} y^{T}(i) R_{1} y(i)
\end{aligned}
$$




$$
\begin{aligned}
& -\left(r_{M}-r_{m}\right) \cdot\left[\sum_{i=k-r_{M}}^{k-r(k)-1} y^{T}(i)\left[R_{2}+W_{2}\right] y(i)+\sum_{i=k-r(k)}^{k-r_{m}-1} y^{T}(i)\left[R_{2}+W_{1}\right] y(i)\right] \\
& -\left(r_{M}-r_{m}\right) \cdot\left\{\sum_{i=k-r_{M}}^{k-r_{m}-1} y^{T}(i) V_{1} y(i)+\sum_{i=k-r_{M}}^{k-r_{m}-1} y^{T}(i) V_{2} y(i)\right\} \\
& -r_{m} \cdot\left[\sum_{i=k-r_{m}}^{k-1} y^{T}(i) R_{3} y(i)\right]-x^{T}\left(k-r_{m}\right)\left[T-\left(r_{M}-r_{m}\right) \cdot W_{1}-S\right] x\left(k-r_{m}\right) \\
& -x^{T}\left(k-r_{M}\right)\left[S+\left(r_{M}-r_{m}\right) \cdot W_{2}\right] x\left(k-r_{M}\right) \\
& -\left(r_{M}-r_{m}\right) \cdot \sum_{i=k-r_{M}}^{k-r(k)-1}\left[\begin{array}{l}
x(i) \\
y(i)
\end{array}\right]^{T}\left[\begin{array}{ll}
Q_{1} & W_{2} \\
* & Q_{2}
\end{array}\right]\left[\begin{array}{l}
x(i) \\
y(i)
\end{array}\right] \\
& -\left(r_{M}-r_{m}\right) \cdot \sum_{i=k-r(k)}^{k-r_{m}-1}\left[\begin{array}{l}
x(i) \\
y(i)
\end{array}\right]^{T}\left[\begin{array}{ll}
Q_{1} & W_{1} \\
* & Q_{2}
\end{array}\right]\left[\begin{array}{l}
x(i) \\
y(i)
\end{array}\right] \\
& +\left[-2 z^{T}(k) w(k)-\gamma \cdot w^{T}(k) w(k)\right] .
\end{aligned}
$$

Without loss of generality, assuming that $0 \leq r_{m}<r(k)<r_{M}$ and $r_{i-1} \leq r(k) \leq r_{i}, i=$ $1,2, \ldots, p$, for some $k$, by Lemma 4 we have the following results:

$$
\begin{aligned}
& -r_{M} \cdot \sum_{i=k-r_{M}}^{k-1} y^{T}(i) R_{1} y(i) \leq-\left[\sum_{i=k-r_{M}}^{k-1} y^{T}(i)\right]^{T} R_{1}\left[\sum_{i=k-r_{M}}^{k-1} y^{T}(i)\right] \\
& =-\left[x(k)-x\left(k-r_{M}\right)\right]^{T} R_{1}\left[x(k)-x\left(k-r_{M}\right)\right], \\
& -\left(r_{M}-r_{m}\right) \cdot\left[\sum_{i=k-r_{M}}^{k-r(k)-1} y^{T}(i)\left[R_{2}+W_{2}\right] y(i)+\sum_{i=k-r(k)}^{k-r_{m}-1} y^{T}(i)\left[R_{2}+W_{1}\right] y(i)\right] \\
& \leq-\frac{\left(r_{M}-r_{m}\right)}{\left(r_{M}-r(k)\right)} \cdot \xi_{1}-\frac{\left(r_{M}-r_{m}\right)}{\left(r(k)-r_{m}\right)} \cdot \xi_{2} \leq-\gamma_{23}^{i} \cdot \xi_{1}-\gamma_{24}^{i} \cdot \xi_{2}, \\
& -r_{m} \cdot \sum_{i=k-r_{m}}^{k-1} y^{T}(i) R_{3} y(i) \leq-\left[x(k)-x\left(k-r_{m}\right)\right]^{T} R_{3}\left[x(k)-x\left(k-r_{m}\right)\right], \\
& -\left(r_{M}-r_{m}\right) \cdot \sum_{i=k-r_{M}}^{k-r_{m}-1} y^{T}(i) V_{1} y(i) \\
& \quad=-\left(r_{M}-r_{m}\right) \cdot\left[\sum_{i=k-r_{M}}^{k-r(k)-1} y^{T}(i) V_{1} y(i)+\sum_{i=k-r(k)}^{k-r_{m}-1} y^{T}(i) V_{1} y(i)\right] \\
& \leq-\frac{\left(r_{M}-r_{m}\right)}{\left(r_{M}-r(k)\right)} \cdot\left[\left(x(k-r(k))-x\left(k-r_{M}\right)\right)^{T} V_{1}\left(x(k-r(k))-x\left(k-r_{M}\right)\right)\right] \\
& \quad-\frac{\left(r_{M}-r_{m}\right)}{\left(r(k)-r_{m}\right)} \cdot\left[\left(x\left(k-r_{m}\right)-x(k-r(k))\right)^{T} V_{1}\left(x\left(k-r_{m}\right)-x(k-r(k))\right)\right], \\
& -\left(r_{M}-r_{m}\right) \cdot \sum_{i=k-r_{M}}^{k-r_{m}-1} y^{T}(i) V_{2} y(i) \\
& \leq-\left[x\left(k-r_{m}\right)-x\left(k-r_{M}\right)\right]^{T} V_{2}\left[x\left(k-r_{m}\right)-x\left(k-r_{M}\right)\right],
\end{aligned}
$$


where

$$
\begin{aligned}
& \xi_{1}=\left[x(k-r(k))-x\left(k-r_{M}\right)\right]^{T}\left(R_{2}+W_{2}\right)\left[x(k-r(k))-x\left(k-r_{M}\right)\right], \\
& \xi_{2}=\left[x\left(k-r_{m}\right)-x(k-r(k))\right]^{T}\left(R_{2}+W_{1}\right)\left[x\left(k-r_{m}\right)-x(k-r(k))\right] .
\end{aligned}
$$

Define

$$
Z^{T}(k)=\left[\begin{array}{lllll}
x^{T}(k) & x^{T}(k-r(k)) & x^{T}\left(k-r_{M}\right) & x^{T}\left(k-r_{m}\right) & w^{T}(k)
\end{array}\right] .
$$

By Park inequality in Lemma 5 with (3b) and (7d) we have

$$
\begin{aligned}
& -\left(r_{M}-r_{m}\right) \cdot \sum_{i=k-r_{M}}^{k-r_{m}-1} y^{T}(i) U_{1} y(i) \\
& \leq-Z^{T}(k)\left[\begin{array}{l}
M_{1} \\
M_{2}
\end{array}\right]^{T}\left[\begin{array}{ll}
V_{1} & X \\
* & V_{1}
\end{array}\right]\left[\begin{array}{c}
M_{1} \\
M_{2}
\end{array}\right] Z(k) \\
& =Z^{T}(k)\left[\begin{array}{ccccc}
0 & 0 & 0 & 0 & 0 \\
* & -2 V_{1}+X+X^{T} & V_{1}-X^{T} & -X+V_{1} & 0 \\
* & * & -V_{1} & X & 0 \\
* & * & * & -V_{1} & 0 \\
* & * & * & * & 0
\end{array}\right] Z(k),
\end{aligned}
$$

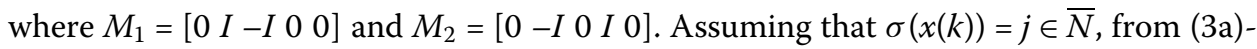
(3c), (6d), and (8a)-(8b) we derive the following result:

$$
\Delta V\left(x_{k}\right)+\left[-2 z^{T}(k) w(k)-\gamma \cdot w^{T}(k) w(k)\right] \leq-x^{T}(k) U_{j} x(k)+Z^{T}(k) \cdot \hat{\Sigma}_{j}^{i} \cdot Z(k),
$$

where

$$
\begin{aligned}
& \hat{\Sigma}_{j}^{i}=\Sigma_{1 j}^{i}-\left[\begin{array}{c}
\bar{\Sigma}_{16 j} \\
\bar{\Sigma}_{26 j} \\
0 \\
0 \\
\bar{\Sigma}_{56 j}
\end{array}\right] \Sigma_{66 j}^{-1}\left[\begin{array}{c}
\bar{\Sigma}_{16 j} \\
\bar{\Sigma}_{26 j} \\
0 \\
0 \\
\bar{\Sigma}_{56 j}
\end{array}\right]^{T}-\left[\begin{array}{c}
\bar{\Sigma}_{17 j} \\
\bar{\Sigma}_{27 j} \\
0 \\
0 \\
\bar{\Sigma}_{57 j}
\end{array}\right] \Sigma_{77 j}^{-1}\left[\begin{array}{c}
\bar{\Sigma}_{17 j} \\
\bar{\Sigma}_{27 j} \\
0 \\
0 \\
\bar{\Sigma}_{57 j}
\end{array}\right]^{T}, \\
& \Sigma_{1 j}^{i}=\left[\begin{array}{ccccc}
\Sigma_{11 j} & 0 & \Sigma_{13 j} & \Sigma_{14 j} & \bar{\Sigma}_{15 j} \\
* & \Sigma_{22 j}^{i} & \Sigma_{23 j}^{i} & \Sigma_{24 j}^{i} & \bar{\Sigma}_{25 j} \\
* & * & \Sigma_{33 j}^{i} & 0 & 0 \\
* & * & * & \Sigma_{44 j}^{i} & 0 \\
* & * & * & * & \bar{\Sigma}_{55 j}
\end{array}\right], \\
& \bar{\Sigma}_{15 j}=-\left(A_{z j}+\Delta A_{z j}\right)^{T}, \quad \bar{\Sigma}_{16 j}=\left(A_{j}+\Delta A_{j}-I\right)^{T} \Theta, \quad \bar{\Sigma}_{17 j}=\left(A_{j}+\Delta A_{j}\right)^{T} P, \\
& \bar{\Sigma}_{25 j}=-\left(B_{z j}+\Delta B_{z j}\right)^{T}, \quad \bar{\Sigma}_{26 j}=\left(B_{j}+\Delta B_{j}\right)^{T} \Theta, \quad \bar{\Sigma}_{27 j}=\left(B_{j}+\Delta B_{j}\right)^{T} P, \\
& \bar{\Sigma}_{55 j}=-\left(D_{z j}+\Delta D_{z j}\right)-\left(D_{z j}+\Delta D_{z j}\right)^{T}-\gamma \cdot I, \\
& \bar{\Sigma}_{56 j}=\left(D_{j}+\Delta D_{j}\right)^{T} \Theta, \quad \bar{\Sigma}_{57 j}=\left(D_{j}+\Delta D_{j}\right)^{T} P,
\end{aligned}
$$

$\Sigma_{k l j}$ and $\Sigma_{k l j}^{i}, k, l=1,2, \ldots, 7$, and $\Theta$ are defined in (3f). 
Define

$$
\bar{\Sigma}_{j}^{i}=\left[\begin{array}{cc}
\Sigma_{1 j}^{i} & \bar{\Sigma}_{2 j} \\
* & \Sigma_{3 j}
\end{array}\right]=\left[\begin{array}{cc}
\Sigma_{1 j}^{i} & \Sigma_{2 j} \\
* & \Sigma_{3 j}
\end{array}\right]+\Pi_{j} \bar{\Delta}_{j}(k) \Psi_{j}^{T}+\Psi_{j} \bar{\Delta}_{j}^{T}(k) \Pi_{j}^{T},
$$

where

$$
\begin{aligned}
& \bar{\Sigma}_{2 j}=\left[\begin{array}{cc}
\bar{\Sigma}_{16 j} & \bar{\Sigma}_{17 j} \\
\bar{\Sigma}_{26 j} & \bar{\Sigma}_{27 j} \\
0 & 0 \\
0 & 0 \\
\bar{\Sigma}_{56 j} & \bar{\Sigma}_{57 j}
\end{array}\right], \quad \Sigma_{2 j}=\left[\begin{array}{cc}
\Sigma_{16 j} & \Sigma_{17 j} \\
\Sigma_{26 j} & \Sigma_{27 j} \\
0 & 0 \\
0 & 0 \\
\Sigma_{56 j} & \Sigma_{57 j}
\end{array}\right], \\
& \Sigma_{3 j}=\left[\begin{array}{cc}
\Sigma_{66 j} & 0 \\
0 & \Sigma_{77 j}
\end{array}\right], \quad \Pi_{j}=\left[\begin{array}{ccccccc}
0 & 0 & 0 & 0 & 0 & \Sigma_{68 j}^{T} & \Sigma_{78 j}^{T} \\
0 & 0 & 0 & 0 & \Sigma_{59 j}^{T} & 0 & 0
\end{array}\right]^{T} \text {, } \\
& \bar{\Delta}_{j}(k)=\left[\begin{array}{cc}
\Delta_{j}(k) & 0 \\
0 & \Delta_{z j}(k)
\end{array}\right]=\left[\begin{array}{cc}
I-\Gamma_{j}(k) \Xi_{j} & 0 \\
0 & I-\Gamma_{z j}(k) \Xi_{z j}
\end{array}\right]^{-1}\left[\begin{array}{cc}
\Gamma_{i}(k) & 0 \\
0 & \Gamma_{z i}(k)
\end{array}\right] \\
& =\left\{\left[\begin{array}{cc}
I & 0 \\
0 & I
\end{array}\right]-\left[\begin{array}{cc}
\Gamma_{j}(k) & 0 \\
0 & \Gamma_{z j}(k)
\end{array}\right]\left[\begin{array}{cc}
\Xi_{j} & 0 \\
0 & \Xi_{z j}
\end{array}\right]\right\}^{-1}\left[\begin{array}{cc}
\Gamma_{i}(k) & 0 \\
0 & \Gamma_{z i}(k)
\end{array}\right], \\
& \Psi_{j}=\left[\begin{array}{ccccccc}
N_{A j} & N_{B j} & 0 & 0 & N_{D j} & 0 & 0 \\
N_{Z A j} & N_{Z B j} & 0 & 0 & N_{Z D j} & 0 & 0
\end{array}\right]^{T} \text {. }
\end{aligned}
$$

By (3d) with Lemma 1 and switching signal in (2c) we have

$$
x^{T}(k) U_{j} x(k) \geq 0, \quad \forall x(k) \in \bar{\Omega}_{j} .
$$

By Lemmas 2 and 3 with (9d), the condition $\Sigma_{j}^{i}<0$ in (3c) implies $\bar{\Sigma}_{j}^{i}<0$ in (9c), which also implies $\hat{\Sigma}_{j}^{i}<0$ in (9b). From (10) by summing (9a) from 0 to $\ell$ we derive the following condition:

$$
V\left(x_{\ell}\right)-V\left(\varphi_{0}\right)+\sum_{k=0}^{\ell}\left[-2 \cdot z^{T}(k) w(k)-\gamma \cdot w^{T}(k) w(k)\right] \leq 0 .
$$

With zero initial condition $\left(\varphi(k)=0,-r_{M} \leq k \leq 0\right)$ we have

$$
V\left(\varphi_{0}\right)=0
$$

By the definition of the functional $V\left(x_{k}\right)$ in (4) we have

$$
V\left(x_{\ell}\right) \geq 0
$$

From the previous derivations the following condition is guaranteed:

$$
-\gamma \cdot \sum_{k=0}^{\ell}\left[w^{T}(k) w(k)\right] \leq 2 \cdot \sum_{k=0}^{\ell}\left[z^{T}(k) w(k)\right] .
$$


By Definition 1 the system (1a)-(1h) is passive by the switching signal designed in (2c). This completes this proof.

Remark 2 In view of (9a) in the proof of Theorem 1, we have $\Delta V\left(x_{k}\right)<0$ when $w(k)=0$. The internal stability of system (1a)-(1h) can be achieved by the proposed results.

Remark 3 The matrix perturbations in (1d)-(1h) are usually called linear fractional perturbations $[11,18,19,32,33]$. The parametric perturbations in $[10,14,15,29,30]$ are the special conditions of system under consideration with $\Xi_{i}=0, \Xi_{z i}=0, i \in \bar{N}$.

Remark 4 The Lyapunov functional proposed in (4) is different from our previous ones in $[18,19]$. In this paper, upper and lower bounds of delay are used instead of interval time-varying $r(k)$ in $[18,19]$. Hence, some complicated derivations can be ignored in this paper. On the other hand, the discrete Jensen inequality approach is used instead of the nonnegative inequality approach in $[18,19]$. Since the number and dimension of variables have been reduced, the LMI program can be formulated easily. In this paper, we also use a delay-partitioning approach to improve the conservativeness of the developed results. Uniform or nonuniform partitions can be performed by our proposed results.

\section{Passive switching control for uncertain discrete switched system}

Next, we consider the passive switching control of the following system:

$$
\begin{aligned}
& x(k+1)=\left[A_{\sigma}+\Delta A_{\sigma}(k)\right] x(k)+\left[B_{\sigma}+\Delta B_{\sigma}(k)\right] x(k-r(k)) \\
& +\left[D_{w \sigma}+\Delta D_{w \sigma}(k)\right] w(k)+\left[D_{u \sigma}+\Delta D_{u \sigma}(k)\right] u(k), \quad k=0,1,2, \ldots, \\
& z(k)=\left[A_{z \sigma}+\Delta A_{z \sigma}(k)\right] x(k)+\left[B_{z \sigma}+\Delta B_{z \sigma}(k)\right] x(k-r(k)) \\
& +\left[D_{z w \sigma}+\Delta D_{z w \sigma}(k)\right] w(k), \quad k=0,1,2, \ldots, \\
& x(\theta)=\varphi(\theta), \quad \theta=-r_{M},-r_{M}+1, \ldots, 0,
\end{aligned}
$$

where $u(k) \in \mathfrak{R}^{v}$ is the control input, $D_{u i}, i=1,2, \ldots, N$, are some given constant matrices of appropriate dimensions. Other definitions are shown in system (1a)-(1h). $\Delta A_{i}(k)$, $\Delta B_{i}(k), \Delta D_{w i}(k), \Delta D_{u i}(k), \Delta A_{z i}(k), \Delta B_{z i}(k)$, and $\Delta D_{z w i}(k)$ are some perturbed matrices satisfying the following conditions:

$$
\begin{aligned}
& {\left[\begin{array}{llll}
\Delta A_{i}(k) & \Delta B_{i}(k) & \Delta D_{w i}(k) & \Delta D_{u i}(k)
\end{array}\right]} \\
& \quad=M_{i} \cdot \Delta_{i}(k) \cdot\left[\begin{array}{llll}
N_{A i} & N_{B i} & N_{D W i} & N_{D U i}
\end{array}\right] \\
& {\left[\begin{array}{llll}
\Delta A_{z i}(k) & \Delta B_{z i}(k) & \Delta D_{z w i}(k)
\end{array}\right]=M_{Z i} \cdot \Delta_{Z i}(k) \cdot\left[\begin{array}{lll}
N_{Z A i} & N_{Z B i} & N_{Z W i}
\end{array}\right],} \\
& \Delta_{i}(k)=\left[\begin{array}{ll}
I-\Gamma_{i}(k) \Xi_{i}
\end{array}\right]^{-1} \Gamma_{i}(k), \quad \Xi_{i} \Xi_{i}^{T}<I, \\
& \Delta_{Z i}(k)=\left[\begin{array}{ll}
I-\Gamma_{z i}(k) \Xi_{z i}
\end{array}\right]^{-1} \Gamma_{z i}(k), \quad \Xi_{z i} \Xi_{z i}^{T}<I,
\end{aligned}
$$

where $M_{i} \in \Re^{n \times q}, M_{Z i} \in \Re^{p \times q}, N_{A i}, N_{B i}, N_{D W i}, N_{D U i}, N_{Z A i}, N_{Z B i}, N_{Z W i}, \Xi_{i}$, and $\Xi_{z i}, i=$ $1,2, \ldots, N$, are some given constant matrices with appropriate dimensions, $\Gamma_{i}(k)$ and $\Gamma_{z i}(k)$ are some unknown matrices satisfying

$$
\Gamma_{i}^{T}(k) \Gamma_{i}(k) \leq I, \quad \Gamma_{z i}^{T}(k) \Gamma_{z i}(k) \leq I .
$$


The switching domains are also defined by

$$
\Omega_{i}\left(U_{i}\right)=\left\{x \in \Re^{n}: x^{T} U_{i} x \geq 0\right\}, \quad i=1,2, \ldots, N,
$$

where the matrices $U_{i}>0, i=1,2, \ldots, N$, will be selected from our developed results, and

$$
\begin{aligned}
& \bar{\Omega}_{1}=\Omega_{1}, \quad \bar{\Omega}_{2}=\Omega_{2} \backslash \bar{\Omega}_{1}, \quad \bar{\Omega}_{3}=\Omega_{3} \backslash \bar{\Omega}_{1} \backslash \bar{\Omega}_{2}, \quad \ldots, \\
& \bar{\Omega}_{N}=\Omega_{N} \backslash \bar{\Omega}_{1} \backslash \ldots \backslash \bar{\Omega}_{N-1} .
\end{aligned}
$$

By the definition of domains the switching signal can be designed by

$$
\sigma(x(k))=i, \quad \forall x(k) \in \bar{\Omega}_{i}
$$

where $\bar{\Omega}_{i}$ is defined in (12b). Now we define the state feedback switching control to achieve the stabilization and passivity for the switched system in (11a)-(11h):

$$
u(k)=-K_{i} x(k), \quad \text { when } \sigma(x(k))=i,
$$

where the state feedback gain $K_{i} \in \Re^{v \times n}$ will be selected from our developed result.

Lemma 6 [41] For matrices $X, Y$, and $Z$ with $X=X^{T}$ and $Z=Z^{T}$, the following statements are equivalent:

(a)

$$
S=\left[\begin{array}{cc}
X & Y \\
* & -Z^{-1}
\end{array}\right]<0 .
$$

(b) There exists a scalar $\eta>0$ such that

$$
\left[\begin{array}{ccc}
X & \eta \cdot Y & 0 \\
* & -2 \eta \cdot I & Z \\
* & * & -Z
\end{array}\right]<0
$$

Lemma 7 [42] Suppose that $\Delta_{i}(k)$ is defined in (11f) and satisfies (11h). Then for real matrices $V_{i}, W_{i}$, and $X_{i}$ with $X_{i}=X_{i}^{T}$, the following statements are equivalent:

(a)

$$
X_{i}+V_{i} \Delta_{i}(k) W_{i}+W_{i}^{T} \Delta_{i}^{T}(k) V_{i}^{T}<0
$$

(b) There exists a scalar $\varepsilon_{i}>0$ such that

$$
\left[\begin{array}{ccc}
X_{i} & \varepsilon_{i} \cdot V_{i} & W_{i}^{T} \\
* & -\varepsilon_{i} \cdot I & \varepsilon_{i} \cdot \Xi_{i}^{T} \\
* & * & -\varepsilon_{i} \cdot I
\end{array}\right]<0,
$$

where the matrix $\Xi_{i}$ is defined in (11f). 
Definition 2 [43] The discrete switched system (11a)-(11h) with switching signal in (12c) and switching control in (13) is called passive if there exists a constant $\gamma>0$ such that

$$
-\gamma \cdot \sum_{k=0}^{\ell} w^{T}(k) w(k) \leq 2 \cdot \sum_{k=0}^{\ell} z^{T}(k) w(k)
$$

for all $\ell>0$ and zero initial condition $x(\theta)=0, \theta=-r_{M},-r_{M}+1, \ldots, 0$. If the parameter $\ell$ is selected as $\infty$, then the disturbance input $w$ belongs to $L_{2}(0, \infty)$.

Theorem 2 For some selected integers $0<r_{m}=r_{0}<r_{1}<r_{2}<\cdots<r_{p-1}<r_{p}=r_{M}$, constants $\gamma>0,0 \leq \alpha_{i} \leq 1, i \in \bar{N}$, and $\sum_{i=1}^{N} \alpha_{i}=1$, system (11a)-(11h) is passive by the designed switching signal in (12c) and switching control in (13) with control gains $K_{i}=\hat{K}_{i} / \eta_{i}$ if there exist $n \times n$ symmetric matrices $P>0, Q_{1}>0, Q_{2}>0, R_{1}>0, R_{2}>0, R_{3}>0, S>0, T>0, V_{1}>0$, $V_{2}>0, U_{j}, W_{1}, W_{2}$, matrices $X \in \Re^{n \times n}, \hat{K}_{j} \in \Re^{v \times n}, j=1,2, \ldots, N$, and constants $\varepsilon_{j}>0$, $\eta_{j}>0, j=1,2, \ldots, N$, such that the following LMI conditions are satisfied:

$$
\begin{aligned}
& R_{2}+W_{1}>0, \quad R_{2}+W_{2}>0, \\
& {\left[\begin{array}{cc}
Q_{1} & W_{1} \\
* & Q_{2}
\end{array}\right]>0, \quad\left[\begin{array}{cc}
Q_{1} & W_{2} \\
* & Q_{2}
\end{array}\right]>0, \quad\left[\begin{array}{cc}
V_{1} & X \\
* & V_{1}
\end{array}\right]>0,} \\
& \tilde{\Sigma}_{j}^{i}=\left[\begin{array}{cc}
\tilde{\Sigma}_{j 1}^{i} & \tilde{\Sigma}_{j 2} \\
* & \tilde{\Sigma}_{j 3}
\end{array}\right]<0, \quad i=1,2, \ldots, p, j=1,2, \ldots, N, \\
& \sum_{i=1}^{N} \alpha_{i} \cdot U_{i}>0,
\end{aligned}
$$

where

$$
\begin{aligned}
\tilde{\Sigma}_{j 1}^{i} & =\left[\begin{array}{ccccc}
\Sigma_{11 j} & 0 & \Sigma_{13 j} & \Sigma_{14 j} & \Sigma_{15 j} \\
* & \Sigma_{22 j}^{i} & \Sigma_{23 j}^{i} & \Sigma_{24 j}^{i} & \Sigma_{25 j} \\
* & * & \Sigma_{33 j}^{i} & \Sigma_{34 j} & 0 \\
* & * & * & \Sigma_{44 j}^{i} & 0 \\
* & * & * & * & \Sigma_{55 j}
\end{array}\right], \\
\tilde{\Sigma}_{j 2} & =\left[\begin{array}{cccccccc}
\Sigma_{16 j} & \Sigma_{17 j} & 0 & 0 & 0 & 0 & \Sigma_{112 j} & \Sigma_{113 j} \\
\Sigma_{26 j} & \Sigma_{27 j} & 0 & 0 & 0 & 0 & \Sigma_{212 j} & \Sigma_{213 j} \\
0 & 0 & 0 & 0 & 0 & 0 & 0 & 0 \\
0 & 0 & 0 & 0 & 0 & 0 & 0 & 0 \\
\Sigma_{56 j} & \Sigma_{57 j} & 0 & 0 & 0 & \Sigma_{511 j} & \Sigma_{512 j} & \Sigma_{513 j}
\end{array}\right], \\
\tilde{\Sigma}_{j 3} & =\left[\begin{array}{cccccccc}
\Sigma_{66 j} & 0 & \Sigma_{68 j} & 0 & \Sigma_{610 j} & 0 & 0 & 0 \\
* & \Sigma_{77 j} & 0 & \Sigma_{79 j} & \Sigma_{710 j} & 0 & 0 & 0 \\
* & * & \Sigma_{88 j} & 0 & 0 & 0 & 0 & 0 \\
* & * & * & \Sigma_{99 j} & 0 & 0 & 0 & 0 \\
* & * & * & * & \Sigma_{1010 j} & 0 & \Sigma_{1012 j} & 0 \\
* & * & * & * & * & \Sigma_{111 j} & 0 & \Sigma_{1113 j} \\
* & * & * & * & * & * & \Sigma_{1212 j} & 0 \\
* & * & * & * & * & * & * & \Sigma_{1313 j}
\end{array}\right],
\end{aligned}
$$




$$
\begin{aligned}
& \Sigma_{11 j}=-P+T+\left(r_{M}-r_{m}\right)^{2} \cdot Q_{1}-R_{1}-R_{3}+U_{j} \\
& \Sigma_{13 j}=R_{1}, \quad \Sigma_{14 j}=R_{3}, \quad \Sigma_{15 j}=-A_{z j}^{T} \text {, } \\
& \Sigma_{16 j}=\eta_{j} \cdot\left(A_{j}-I\right)^{T}-\hat{K}_{j}^{T} D_{u j}^{T}, \quad \Sigma_{17 j}=\eta_{j} \cdot A_{j}^{T}-\hat{K}_{j}^{T} D_{u j}^{T}, \\
& \Sigma_{112 j}=\eta_{j} \cdot N_{A j}^{T}-\hat{K}_{j}^{T} N_{D U j}^{T}, \quad \Sigma_{113 j}=N_{Z A j}^{T} \text {, } \\
& \gamma_{23}^{i}=\frac{\left(r_{M}-r_{m}\right)}{\left(r_{M}-r_{i-1}\right)}, \quad \gamma_{24}^{i}=\frac{\left(r_{M}-r_{m}\right)}{\left(r_{i}-r_{m}\right)} \\
& \Sigma_{22 j}^{i}=-\gamma_{23}^{i} \cdot\left(R_{2}+W_{2}\right)-\gamma_{24}^{i} \cdot\left(R_{2}+W_{1}\right)-\left(r_{M}-r_{m}\right) \cdot\left(W_{1}-W_{2}\right)-2 V_{1}+X+X^{T} \text {, } \\
& \Sigma_{23 j}^{i}=\gamma_{23}^{i} \cdot\left(R_{2}+W_{2}\right)+V_{1}-X^{T}, \quad \Sigma_{24}^{i}=\gamma_{24}^{i} \cdot\left(R_{2}+W_{1}\right)-X+V_{1}, \\
& \Sigma_{25 j}=-B_{z j}^{T}, \quad \Sigma_{26 j}=\eta_{j} \cdot B_{j}^{T}, \quad \Sigma_{27 j}=\eta_{j} \cdot B_{j}^{T}, \\
& \Sigma_{212 j}=\eta_{j} \cdot N_{B j}^{T}, \quad \Sigma_{213 j}=N_{Z B j}^{T}, \\
& \Sigma_{33 j}^{i}=-S-R_{1}-\gamma_{23}^{i} \cdot\left(R_{2}+W_{2}\right)-\left(r_{M}-r_{m}\right) \cdot W_{2}-V_{1}-V_{2}, \quad \Sigma_{34 j}=V_{2}+X, \\
& \Sigma_{44 j}^{i}=-(T-S)-\gamma_{24}^{i} \cdot\left(R_{2}+W_{1}\right)-R_{3}+\left(r_{M}-r_{m}\right) \cdot W_{1}-V_{1}-V_{2}, \\
& \Sigma_{55 j}=-D_{z w j}-D_{z w j}^{T}-\gamma \cdot I, \quad \Sigma_{56 j}=\eta_{j} \cdot D_{w j}^{T}, \quad \Sigma_{57 j}=\eta_{j} \cdot D_{w j}^{T}, \\
& \Sigma_{511 j}=-\varepsilon_{j} \cdot M_{Z j}, \quad \Sigma_{512 j}=\eta_{j} \cdot N_{D W j}^{T}, \quad \Sigma_{513 j}=N_{Z W j}^{T}, \quad \Sigma_{66 j}=\Sigma_{77 j}=-2 \eta_{j} \cdot I \text {, } \\
& \Theta=r_{M}^{2} \cdot R_{1}+\left(r_{M}-r_{m}\right)^{2}\left(Q_{2}+R_{2}+V_{1}+V_{2}\right)+r_{m}^{2} \cdot R_{3}, \\
& \Sigma_{68 j}=\Theta, \quad \Sigma_{610 j}=\varepsilon_{j} \cdot M_{j}, \quad \Sigma_{79 j}=P, \\
& \Sigma_{710 j}=\varepsilon_{j} \cdot M_{j}, \quad \Sigma_{88 j}=-\Theta, \quad \Sigma_{99 j}=-P, \\
& \Sigma_{1010 j}=\Sigma_{1111 j}=\Sigma_{1212 j}=\Sigma_{1313 j}=-\varepsilon_{j} \cdot I, \\
& \Sigma_{1012 j}=\varepsilon_{j} \cdot \Xi_{j}^{T}, \quad \Sigma_{1113 j}=\varepsilon_{j} \cdot \Xi_{z j}^{T} .
\end{aligned}
$$

Proof For the functional given in (4), the derivations in (4)-(7f) with (11a)-(13) can be formulated as

$$
\begin{aligned}
& \Delta V\left(x_{k}\right)+\left(r_{M}-r_{m}\right) \cdot\left(\lambda_{1}+\lambda_{2}\right)+\left[-2 z^{T}(k) w(k)-\gamma \cdot w^{T}(k) w(k)\right] \\
& \quad \leq-x^{T}(k) U_{j} x(k)+Z^{T}(k) \cdot \hat{\Sigma}_{j}^{i} \cdot Z(k),
\end{aligned}
$$

where $Z(k)$ is defined in $(8 \mathrm{a})$, and

$$
\begin{aligned}
\hat{\Sigma}_{j}^{i} & =\hat{\Sigma}_{1 j}^{i}-\left[\begin{array}{c}
\hat{\Sigma}_{16 j} \\
\hat{\Sigma}_{26 j} \\
0 \\
0 \\
\hat{\Sigma}_{56 j}
\end{array}\right] \Sigma_{66 j}^{-1}\left[\begin{array}{c}
\hat{\Sigma}_{16 j} \\
\hat{\Sigma}_{26 j} \\
0 \\
0 \\
\hat{\Sigma}_{56 j}
\end{array}\right]^{T}-\left[\begin{array}{c}
\hat{\Sigma}_{17 j} \\
\hat{\Sigma}_{27 j} \\
0 \\
0 \\
\hat{\Sigma}_{57 j}
\end{array}\right] \Sigma_{77 j}^{-1}\left[\begin{array}{c}
\hat{\Sigma}_{17 j} \\
\hat{\Sigma}_{27 j} \\
0 \\
0 \\
\hat{\Sigma}_{57 j}
\end{array}\right]^{T}, \\
\hat{\Sigma}_{1 j}^{i} & =\left[\begin{array}{ccccc}
\Sigma_{11 j} & 0 & \Sigma_{13 j} & \Sigma_{14 j} & \hat{\Sigma}_{15 j} \\
* & \Sigma_{22 j}^{i} & \Sigma_{23 j}^{i} & \Sigma_{24 j}^{i} & \hat{\Sigma}_{25 j} \\
* & * & \Sigma_{33 j}^{i} & 0 & 0 \\
* & * & * & \Sigma_{44 j}^{i} & 0 \\
* & * & * & * & \hat{\Sigma}_{55 j}
\end{array}\right]
\end{aligned}
$$




$$
\begin{aligned}
& \hat{\Sigma}_{15 j}=-\left(A_{z j}+\Delta A_{z j}\right)^{T}, \quad \hat{\Sigma}_{16 j}=\left(A_{j}+\Delta A_{j}-\left(D_{u j}+\Delta D_{u j}\right) K_{j}-I\right)^{T}, \\
& \hat{\Sigma}_{17 j}=\left(A_{j}+\Delta A_{j}-\left(D_{u j}+\Delta D_{u j}\right) K_{j}\right)^{T}, \quad \hat{\Sigma}_{25 j}=-\left(B_{z j}+\Delta B_{z j}\right)^{T}, \\
& \hat{\Sigma}_{26 j}=\left(B_{j}+\Delta B_{j}\right)^{T}, \quad \hat{\Sigma}_{27 j}=\left(B_{j}+\Delta B_{j}\right)^{T}, \\
& \hat{\Sigma}_{55 j}=-\left(D_{z w j}+\Delta D_{z w j}\right)-\left(D_{z w j}+\Delta D_{z w j}\right)^{T}-\gamma \cdot I, \quad \hat{\Sigma}_{56 j}=\left(D_{w j}+\Delta D_{w j}\right)^{T}, \\
& \hat{\Sigma}_{57 j}=\left(D_{w j}+\Delta D_{w j}\right)^{T}, \quad \Sigma_{66 j}=-\Theta^{-1}, \quad \Sigma_{77 j}=-P^{-1},
\end{aligned}
$$

$\Sigma_{k l j}, k, l=1,2, \ldots, 4$ and $\Theta$ are defined in (14c).

Define

$$
\tilde{\Sigma}_{j}^{i}=\left[\begin{array}{cc}
\hat{\Sigma}_{1 j}^{i} & \hat{\Sigma}_{2 j} \\
* & -\hat{\Sigma}_{4 j}^{-1}
\end{array}\right]
$$

where $\hat{\Sigma}_{1 j}^{i}$ is defined in (15c), and

$$
\hat{\Sigma}_{2 j}=\left[\begin{array}{cc}
\hat{\Sigma}_{16 j} & \hat{\Sigma}_{17 j} \\
\hat{\Sigma}_{26 j} & \hat{\Sigma}_{27 j} \\
0 & 0 \\
0 & 0 \\
\hat{\Sigma}_{56 j} & \hat{\Sigma}_{57 j}
\end{array}\right], \quad \hat{\Sigma}_{4 j}=\left[\begin{array}{cc}
-\Sigma_{66 j} & 0 \\
0 & -\Sigma_{77 j}
\end{array}\right]=\left[\begin{array}{cc}
\Theta & 0 \\
0 & P
\end{array}\right] .
$$

Consider the following matrices with constants $\eta_{j}>0, j=1,2, \ldots, N$ :

$$
\tilde{\tilde{\Sigma}}_{j}^{i}=\left[\begin{array}{ccc}
\hat{\Sigma}_{1 j}^{i} & \eta_{j} \cdot \hat{\Sigma}_{2 j} & 0 \\
* & \Sigma_{3 j} & \hat{\Sigma}_{4 j} \\
* & * & -\hat{\Sigma}_{4 j}
\end{array}\right]=\left[\begin{array}{ccc}
\Sigma_{1 j}^{i} & \Sigma_{2 j} & 0 \\
* & \Sigma_{3 j} & \Sigma_{4 j} \\
* & * & -\Sigma_{4 j}
\end{array}\right]+\Pi_{j} \hat{\Delta}_{j}(k) \Psi_{j}^{T}+\Psi_{j} \hat{\Delta}_{j}^{T}(k) \Pi_{j}^{T},
$$

where $\Sigma_{k l j}, k, l=1,2, \ldots, 7$, are defined in (14c),

$$
\begin{aligned}
& \Sigma_{1 j}^{i}=\left[\begin{array}{ccccc}
\Sigma_{11 j} & 0 & \Sigma_{13 j} & \Sigma_{14 j} & \Sigma_{15 j} \\
* & \Sigma_{22 j}^{i} & \Sigma_{23 j}^{i} & \Sigma_{24 j}^{i} & \Sigma_{25 j} \\
* & * & \Sigma_{33 j}^{i} & 0 & 0 \\
* & * & * & \Sigma_{44 j}^{i} & 0 \\
* & * & * & * & \Sigma_{55 j}
\end{array}\right], \quad \Sigma_{2 j}=\left[\begin{array}{cc}
\Sigma_{16 j} & \Sigma_{17 j} \\
\Sigma_{26 j} & \Sigma_{27 j} \\
0 & 0 \\
0 & 0 \\
\Sigma_{56 j} & \Sigma_{57 j}
\end{array}\right], \\
& \Sigma_{3 j}=\left[\begin{array}{cc}
-2 \cdot \eta_{j} \cdot I & 0 \\
0 & -2 \cdot \eta_{j} \cdot I
\end{array}\right], \quad \Sigma_{4 j}=\left[\begin{array}{cc}
\Theta & 0 \\
0 & P
\end{array}\right] \text {, } \\
& \Pi_{j}=\left[\begin{array}{ccccccccc}
0 & 0 & 0 & 0 & 0 & M_{j}^{T} & M_{j}^{T} & 0 & 0 \\
0 & 0 & 0 & 0 & -M_{Z j}^{T} & 0 & 0 & 0 & 0
\end{array}\right]^{T} \text {, } \\
& \hat{\Delta}_{j}(k)=\left[\begin{array}{cc}
\Delta_{j}(k) & 0 \\
0 & \Delta_{z j}(k)
\end{array}\right]=\left[\begin{array}{cc}
I-\Gamma_{j}(k) \Xi_{j} & 0 \\
0 & I-\Gamma_{z j}(k) \Xi_{z j}
\end{array}\right]^{-1}\left[\begin{array}{cc}
\Gamma_{i}(k) & 0 \\
0 & \Gamma_{z i}(k)
\end{array}\right] \\
& =\left\{\left[\begin{array}{cc}
I & 0 \\
0 & I
\end{array}\right]-\left[\begin{array}{cc}
\Gamma_{j}(k) & 0 \\
0 & \Gamma_{z j}(k)
\end{array}\right]\left[\begin{array}{cc}
\Xi_{j} & 0 \\
0 & \Xi_{z j}
\end{array}\right]\right\}^{-1}\left[\begin{array}{cc}
\Gamma_{i}(k) & 0 \\
0 & \Gamma_{z i}(k)
\end{array}\right] \text {, }
\end{aligned}
$$




$$
\Psi_{j}=\left[\begin{array}{ccccccccc}
\eta_{j} \cdot N_{A j}-N_{D L j} \hat{K}_{j} & \eta_{j} \cdot N_{B j} & 0 & 0 & \eta_{j} \cdot N_{D W j} & 0 & 0 & 0 & 0 \\
N_{Z A j} & N_{Z B j} & 0 & 0 & N_{Z W j} & 0 & 0 & 0 & 0
\end{array}\right]^{T}, \quad \hat{K}_{j}=\eta_{j} \cdot K_{j} .
$$

By Lemmas 6 and 7 the condition in (14c) should be imposed to achieve the passivity of system under consideration.

\section{Illustrative examples}

Example 1 We consider system (1a)-(1h) with the following parameters:

$$
\begin{aligned}
& A_{1}=\left[\begin{array}{cc}
1.01 & 0.01 \\
0.01 & 0
\end{array}\right], \quad A_{2}=\left[\begin{array}{cc}
0 & 0.01 \\
0.01 & 1.01
\end{array}\right], \\
& B_{1}=\left[\begin{array}{cc}
-0.1 & 0.1 \\
0 & -0.1
\end{array}\right], \quad B_{2}=\left[\begin{array}{cc}
-0.1 & 0 \\
0.1 & 0.1
\end{array}\right] \text {, } \\
& D_{1}=\left[\begin{array}{cc}
0.1 & 0 \\
0 & 0.1
\end{array}\right], \quad D_{2}=\left[\begin{array}{cc}
0.1 & 0 \\
0.1 & 0.1
\end{array}\right] \\
& A_{z 1}=\left[\begin{array}{cc}
0.1 & 0 \\
0 & 0.2
\end{array}\right], \quad A_{z 2}=\left[\begin{array}{cc}
0.1 & 0 \\
0 & 0.05
\end{array}\right] \text {, } \\
& B_{z 1}=\left[\begin{array}{cc}
0.02 & 0 \\
0 & 0.01
\end{array}\right], \quad B_{z 2}=\left[\begin{array}{cc}
0.01 & 0 \\
0 & 0.02
\end{array}\right] \text {, } \\
& D_{z 1}=\left[\begin{array}{cc}
0.2 & 0.1 \\
0 & 0.2
\end{array}\right], \quad D_{z 2}=\left[\begin{array}{cc}
0.2 & 0 \\
0 & 0.2
\end{array}\right] \text {, } \\
& M_{1}=M_{2}=\left[\begin{array}{cc}
0.1 & 0 \\
0 & 0.1
\end{array}\right], \quad M_{z 1}=M_{z 2}=\left[\begin{array}{cc}
0.05 & 0 \\
0 & 0.1
\end{array}\right] \text {, } \\
& N_{A 1}=N_{A 2}=\left[\begin{array}{cc}
0.02 & 0 \\
0 & 0.01
\end{array}\right], \quad N_{B 1}=N_{B 2}=\left[\begin{array}{cc}
0.01 & 0 \\
0 & 0.02
\end{array}\right] \text {, } \\
& N_{D 1}=N_{D 2}=\left[\begin{array}{cc}
0.01 & 0 \\
0 & 0.01
\end{array}\right], \quad N_{Z A 1}=N_{Z A 2}=N_{B 1} \text {, } \\
& N_{Z B 1}=N_{Z B 2}=N_{A 1}, \quad N_{Z D 1}=N_{Z D 2}=N_{D 1}, \quad \Xi_{1}=\Xi_{2}=\Xi_{z 1}=\Xi_{z 2}=0.01 \cdot I .
\end{aligned}
$$

With $p=2, r_{m}=r_{0}=1, r_{1}=3, r_{M}=r_{2}=4, \gamma=0.5$, and $\alpha_{1}=\alpha_{2}=0.5$, the LMI conditions in Theorem 1 have a feasible solution with (some matrix solutions for LMI variables are not listed here)

$$
U_{1}=\left[\begin{array}{cc}
-0.5249 & -0.0165 \\
-0.0165 & 0.7641
\end{array}\right], \quad U_{2}=\left[\begin{array}{cc}
0.5225 & 0.0166 \\
0.0166 & -0.7635
\end{array}\right] \text {. }
$$

System (1a)-(1h) with (17) is passive with $r_{m}=1, r_{M}=4, \gamma=0.5$ by the switching signal designed by

$$
\sigma= \begin{cases}1, & x \in \bar{\Omega}_{1} \\ 2, & x \in \mathfrak{R}^{2} \backslash \bar{\Omega}_{1}\end{cases}
$$


Table 1 The obtained results for our proposed results

\begin{tabular}{|c|c|c|}
\hline \multicolumn{3}{|c|}{ The delay upper bound and switching domains to guarantee the passivity property of systems } \\
\hline Results & & $\begin{array}{l}\text { Number for } \\
\text { elements of } \\
\text { LMI variables }\end{array}$ \\
\hline [10] & $\begin{array}{l}r_{m}=1, r_{M}=3 \text { fail to guarantee stability even when } \boldsymbol{\Xi}_{i}=0, \\
\boldsymbol{\Xi}_{z i}=0, i=1,2\end{array}$ & Fail \\
\hline [13] & $\begin{array}{l}r_{m}=1, r_{M}=3 \text { cannot design a switching signal to } \\
\text { guarantee the stability even when } \boldsymbol{\Xi}_{i}=0, \boldsymbol{\Xi}_{z i}=0, i=1,2\end{array}$ & Fail \\
\hline [14] & $\begin{array}{l}r_{m}=1, r_{M}=3 \text { fail to guarantee the stability even when } \\
\boldsymbol{\Xi}_{i}=0, \boldsymbol{\Xi}_{z i}=0, i=1,2\end{array}$ & Fail \\
\hline [18] & $\begin{array}{l}r_{m}=1, r_{M}=3 \\
\bar{\Omega}_{1}=\left\{\left[x_{1} x_{2}\right]^{T}: 1.0102 x_{1}^{2}+0.0016 x_{1} x_{2}-0.9057 x_{2}^{2} \leq 0\right\} \\
\bar{\Omega}_{2}=\Re^{2} \backslash \bar{\Omega}_{1} \\
r_{m}=2, r_{M}=4 \\
\bar{\Omega}_{1}=\left\{\left[x_{1} x_{2}\right]^{T}: 0.3961 x_{1}^{2}-0.0012 x_{1} x_{2}-0.4515 x_{2}^{2} \leq 0\right\} \\
\bar{\Omega}_{2}=\Re^{2} \backslash \bar{\Omega}_{1}\end{array}$ & $\begin{array}{l}338 \text { (program } \\
\text { running time } \\
\text { about } 2 \text { minutes) }\end{array}$ \\
\hline $\begin{array}{l}\text { Results of } \\
\text { this paper }\end{array}$ & $\begin{array}{l}r_{m}=1, r_{M}=4\left(p=2, r_{1}=3, \alpha_{1}=\alpha_{2}=0.5\right) \\
\bar{\Omega}_{1}=\left\{\left[x_{1} x_{2}\right]^{T}:-0.5249 x_{1}^{2}-0.033 x_{1} x_{2}+0.7641 x_{2}^{2} \geq 0\right\} \\
\bar{\Omega}_{2}=\Re^{2} \backslash \bar{\Omega}_{1}\end{array}$ & $\begin{array}{l}45 \text { (program } \\
\text { running time } \\
\text { about } 5 \text { seconds) }\end{array}$ \\
\hline
\end{tabular}

where $\bar{\Omega}_{1}=\left\{\left[x_{1} x_{2}\right]^{T}:-0.5249 x_{1}^{2}-0.033 x_{1} x_{2}+0.7641 x_{2}^{2} \geq 0\right\}$. Some delay upper bounds and switching domains in (18) that guarantee the passivity property $(\gamma=0.5)$ for system (1a)-(1h) with (17) are provided in Table 1 for $\alpha_{1}=\alpha_{2}=0.5$.

Two issues about passivity analysis of switched systems:

1. Under arbitrary switching signal, passivity analysis and passive control can be investigated to guarantee the performance for uncertain discrete switched systems.

2. Design of a switching signal (and a switching control) guarantees the passivity property for uncertain discrete switched systems. This paper is focused on this issue. Note that the matrices $A_{1}$ and $A_{2}$ in this example are not Hurwitz (have at least one eigenvalue greater than 1 ) and the results in $[10,13,14]$ cannot be applied to find any feasible solution to guarantee the stability for discrete switching systems for any arbitrary switching.

Example 2 We consider system (11a)-(11h) with the following parameters:

$$
\begin{array}{ll}
A_{1}=\left[\begin{array}{ll}
1.01 & 0.02 \\
0.01 & 0.01
\end{array}\right], & A_{2}=\left[\begin{array}{ll}
0.01 & 0.01 \\
0.02 & 1.01
\end{array}\right], \\
B_{1}=\left[\begin{array}{cc}
-0.2 & 0.1 \\
0 & -0.2
\end{array}\right], & B_{2}=\left[\begin{array}{ll}
0.2 & 0.1 \\
0.1 & 0.1
\end{array}\right], \\
D_{w 1}=\left[\begin{array}{cc}
0.1 & 0.1 \\
0 & 0.1
\end{array}\right], & D_{w 2}=\left[\begin{array}{cc}
0.1 & 0 \\
0.1 & 0.1
\end{array}\right], \\
D_{u 1}=\left[\begin{array}{cc}
1 & 0.2 \\
0.1 & 1
\end{array}\right], & D_{u 2}=\left[\begin{array}{cc}
1 & 0.1 \\
0.2 & 1
\end{array}\right], \\
A_{z 1}=\left[\begin{array}{cc}
0.1 & 0 \\
0 & 0.2
\end{array}\right], & A_{z 2}=\left[\begin{array}{cc}
0.1 & 0 \\
0.1 & 0.1
\end{array}\right], \\
B_{z 1}=\left[\begin{array}{cc}
0.04 & 0 \\
0 & 0.03
\end{array}\right], & B_{z 2}=\left[\begin{array}{cc}
0.1 & 0 \\
0 & 0.1
\end{array}\right],
\end{array}
$$




$$
\begin{aligned}
& D_{z w 1}=\left[\begin{array}{cc}
0.2 & 0.1 \\
0 & 0.2
\end{array}\right], \quad D_{z w 2}=\left[\begin{array}{cc}
0.2 & 0 \\
0 & 0.2
\end{array}\right], \quad M_{1}=M_{2}=\left[\begin{array}{cc}
0.1 & 0 \\
0 & 0.1
\end{array}\right], \\
& M_{z 1}=M_{z 2}=\left[\begin{array}{cc}
0.05 & 0 \\
0 & 0.05
\end{array}\right], \quad N_{A 1}=N_{A 2}=\left[\begin{array}{cc}
0.02 & 0 \\
0 & 0.01
\end{array}\right], \\
& N_{B 1}=N_{B 2}=\left[\begin{array}{cc}
0.01 & 0 \\
0 & 0.02
\end{array}\right], \quad N_{D W 1}=N_{D W 2}=\left[\begin{array}{cc}
0.01 & 0 \\
0 & 0.02
\end{array}\right], \\
& N_{D U 1}=N_{D U 2}=\left[\begin{array}{cc}
0.02 & 0 \\
0 & 0.01
\end{array}\right], \quad N_{Z A 1}=N_{Z A 2}=N_{A 1}, \quad N_{Z B 1}=N_{Z B 2}=N_{B 1}, \\
& N_{Z W 1}=N_{Z W 2}=N_{D W 1}, \quad \Xi_{1}=\Xi_{2}=\Xi_{z 1}=\Xi_{z 2}=0.01 \cdot I .
\end{aligned}
$$

With $p=2, r_{m}=1, r_{1}=4, r_{M}=8, \gamma=1.5$, and $\alpha_{1}=\alpha_{2}=0.5$, the LMI conditions in Theorem 2 have a feasible solution with (some matrix solutions for LMI variables are not listed here)

$$
\begin{array}{ll}
U_{1}=\left[\begin{array}{cc}
0.0056 & 0.0021 \\
0.0021 & -0.0055
\end{array}\right], & U_{2}=\left[\begin{array}{cc}
-0.0036 & -0.0048 \\
-0.0048 & 0.0102
\end{array}\right], \\
\hat{K}_{1}=\left[\begin{array}{cc}
0.8955 & 0.2065 \\
-0.0729 & -0.8734
\end{array}\right], & \hat{K}_{2}=\left[\begin{array}{cc}
-0.7817 & -0.0618 \\
0.1892 & 0.929
\end{array}\right], \\
\eta_{1}=1.7259, & \eta_{2}=1.6971 .
\end{array}
$$

System (1a)-(1h) with (19) is passive with $r_{m}=1, r_{M}=10, \gamma=1.5$ by the switching signal given in (12c) with

$$
\sigma= \begin{cases}1, & x \in \bar{\Omega}_{1} \\ 2, & x \in \bar{\Omega}_{2}\end{cases}
$$

where

$$
\begin{aligned}
& \bar{\Omega}_{1}=\left\{\left[\begin{array}{ll}
x_{1} & x_{2}
\end{array}\right]^{T} \in R^{2}: 0.0056 x_{1}^{2}+0.0042 x_{1} x_{2}-0.0055 x_{2}^{2} \geq 0\right\} \quad \text { and } \\
& \bar{\Omega}_{2}=\Re^{2} \backslash \bar{\Omega}_{1} .
\end{aligned}
$$

The proposed switching control gains in (13) are given by

$$
K_{1}=\hat{K}_{1} / \eta_{1}=\left[\begin{array}{cc}
0.5188 & 0.1196 \\
-0.0422 & -0.5061
\end{array}\right], \quad K_{2}=\hat{K}_{2} / \eta_{2}=\left[\begin{array}{cc}
-0.4606 & -0.0364 \\
0.1115 & 0.5474
\end{array}\right] \text {. }
$$

Under the disturbance inputs $w(k)=\left[10 \times(0.9)^{k}-10 \times(0.4)^{k}\right]^{T}$ shown in Figure 1 and zero initial conditions, the regulated outputs $z(k) \in \mathfrak{R}^{2}$ of switched system (1a)-(1h) with (19)-(21) and no perturbations are shown in Figure 2. Under zero disturbance, the initial state function $\varphi(\theta)=[10-5]^{T}, \theta=-8,-7, \ldots,-1,0$, and no perturbations, the state trajectories $x(k) \in \mathfrak{R}^{2}$ of switched system (1a)-(1h) with (19)-(21) are shown in Figure 3. By Theorem 2 system (1a)-(1h) with (20) and $1 \leq r(k) \leq 8$ is passive by the proposed switching signal in (20) and switching control in (13) with control gains in (21). With $1 \leq r(k) \leq 8$, 


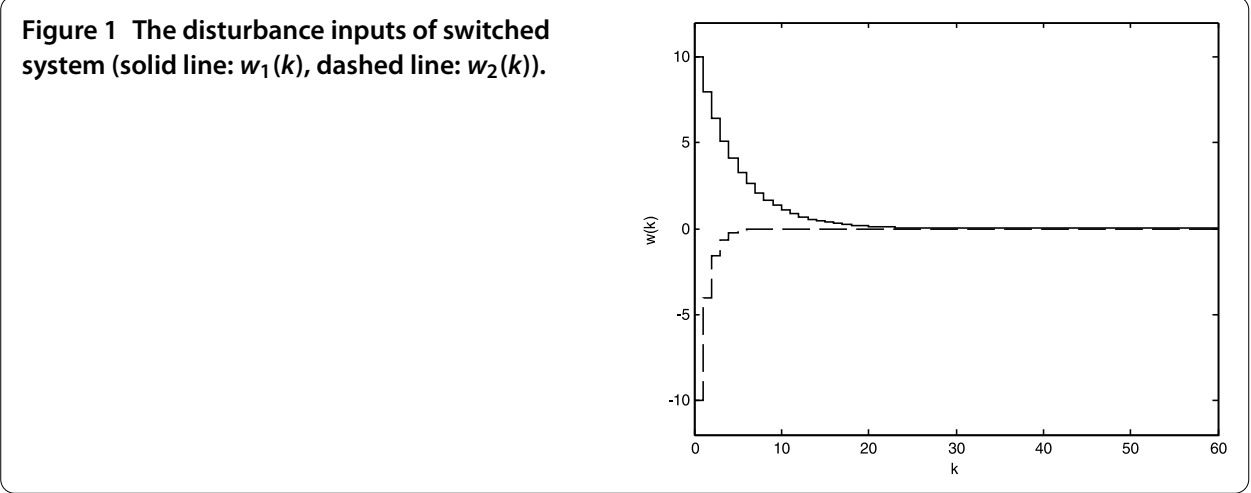

Figure 2 Regulated outputs of switched system (solid line: $z_{1}(k)$, dashed line: $z_{2}(k)$ ).

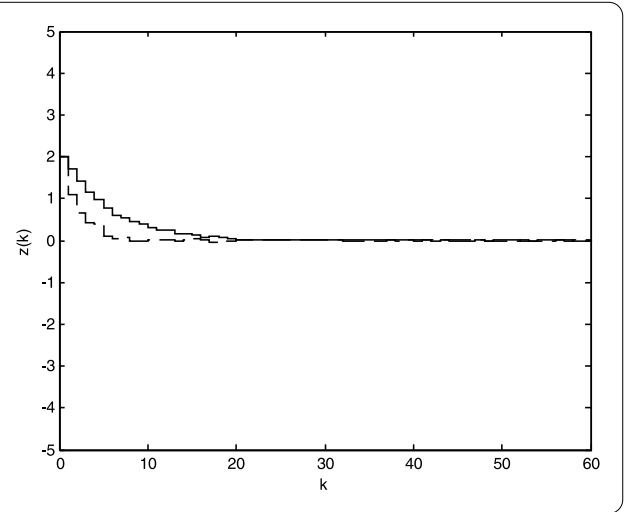

Figure 3 State trajectories for switched system (solid line: $x_{1}(k)$, dashed line: $x_{2}(k)$ ).

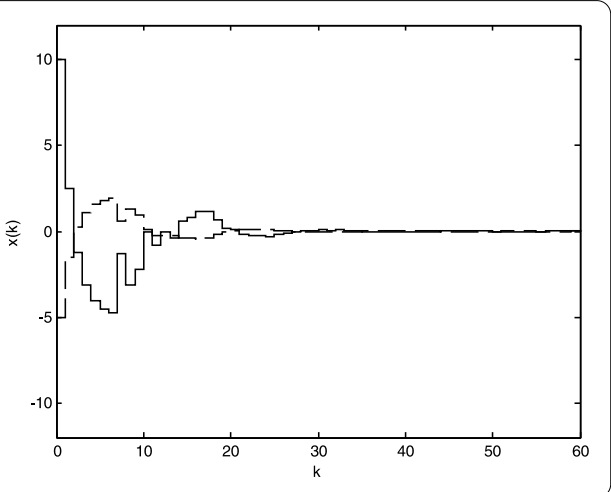

our previous results in $[10,11]$ cannot provide or guarantee any performance of uncertain discrete switched time-delay system. By using Theorem 2, the maximal delay upper bounds with respect to $\gamma=1.5$ and $\gamma=0.1$ that guarantee the passivity property for system (1a)-(1h) with (19) are provided in Table 2 for $\alpha_{1}=\alpha_{2}=0.5, p=2, r_{1}=4$, respectively. The obtained result for passive switching control in this paper is more efficient than our previous results in [11].

There are some major contributions:

(a) Less LMI variable elements are used in this proposed approach. Implementation of LMI program can be achieved easily.

(b) Under the same passivity requirement, a more spacious range of interval time-varying delay can be guaranteed by the proposed approach. 
Table 2 The obtained results for our proposed results

\begin{tabular}{|c|c|c|c|c|}
\hline \multicolumn{5}{|c|}{ The delay upper bound to guarantee the stability and passivity property of systems } \\
\hline \multirow[t]{2}{*}{ [10] } & \multirow[t]{2}{*}{ [11] } & \multicolumn{3}{|c|}{ (switching signal + switching control) $r_{m}=1$} \\
\hline & & Conditions & [19] & $\begin{array}{l}\text { Results of } \\
\text { this paper }\end{array}$ \\
\hline $\begin{array}{l}\text { Fail (stability } \\
\text { for arbitrary } \\
\text { switching) }\end{array}$ & $\begin{array}{l}r_{m}=1, r_{M}=2 \\
(\text { stability for switching } \\
\text { signal design) }\end{array}$ & $\begin{array}{l}\gamma=1.5 \\
\gamma=0.1 \\
\text { Number for } \\
\text { elements of } \\
\text { LMI variables }\end{array}$ & $\begin{array}{l}r_{M}=7 \\
r_{M}=6 \\
348\end{array}$ & $\begin{array}{l}r_{M}=8 \\
r_{M}=7 \\
55\end{array}$ \\
\hline
\end{tabular}

(c) The proposed new Lyapunov functional does not depend on time-varying delay $r(k)$, and some conservativeness can be reduced.

(d) A simple design scheme for the proposed switching signal can be easily generalized to continuous switched time-delay systems.

(e) Better passivity of switching systems can be achieved by switching signal design and switching state feedback control.

(f) The delay-partition approach and Jensen-Park inequalities are used to reduce the conservativeness of the proposed results.

Example 3 The following water quality model is presented in Chapter 11 of [1]:

$$
\begin{aligned}
& x(k+1)=A_{\sigma} x(k)+B_{\sigma} x(k-r(k))+D_{\sigma} w(k), \\
& z(k)=A_{z \sigma} x(k)+B_{z \sigma} x(k-r(k))+D_{z \sigma} w(k),
\end{aligned}
$$

where the time delay $r(k)>0$ is shown to reflect the mixing effect of biochemical constituents in time instance $k$, the state vector $x(k) \in \Re^{n}$ is the water-quality constituents (like algae, ammonia nitrogen, dissolved oxygen, biochemical oxygen demand), the output $z(k) \in \mathfrak{R}^{q}$ is the performance, the disturbance input $w(k) \in \mathfrak{R}^{q}$ is the irregular discharge of effluents and belongs to $L_{2}(0, \infty)$, and the switching signal $\sigma$ is located in the finite set $\{1,2, \ldots, N\}$. Typically, the switching rule $\sigma$ is not known a priori, but we assume that its instantaneous value is available in real time for practical implementations by water pollution management. In this paper, the passivity problem defined in Definition 1 will be investigated. The time-varying delay is defined by $0<r_{m} \leq r(k) \leq r_{M}$, where $r_{m}$ and $r_{M}$ present the extreme cases of light and heavy waste dump loadings, respectively. Consider the following parameters of the model $(N=3)$ :

$$
\begin{aligned}
& A_{1}=\left[\begin{array}{cc}
0.2 & 0.1 \\
0 & 0.2
\end{array}\right], \quad A_{2}=\left[\begin{array}{ll}
0.1 & 0.2 \\
0.2 & 0.1
\end{array}\right], \\
& A_{3}=\left[\begin{array}{cc}
0.1 & 0.1 \\
0.1 & 0.71
\end{array}\right], \quad B_{1}=\left[\begin{array}{cc}
0.2 & 0 \\
0.1 & 0.1
\end{array}\right], \\
& B_{2}=\left[\begin{array}{cc}
-0.2 & 0.1 \\
0 & -0.2
\end{array}\right], \quad B_{3}=\left[\begin{array}{cc}
0.2 & 0 \\
0 & 0.3
\end{array}\right], \\
& D_{1}=\left[\begin{array}{c}
0.05 \\
0.1
\end{array}\right], \quad D_{2}=\left[\begin{array}{c}
0.1 \\
0.05
\end{array}\right], \quad D_{3}=\left[\begin{array}{l}
0.1 \\
0.1
\end{array}\right],
\end{aligned}
$$




$$
\begin{aligned}
& A_{z 1}=\left[\begin{array}{ll}
0.1 & 0.3
\end{array}\right], \quad A_{z 2}=\left[\begin{array}{ll}
0.2 & 0.2
\end{array}\right], \\
& A_{z 3}=\left[\begin{array}{ll}
0.1 & 0.3
\end{array}\right], \quad B_{z 1}=\left[\begin{array}{ll}
0.2 & 0.2
\end{array}\right], \\
& B_{z 2}=\left[\begin{array}{ll}
0.1 & 0.2
\end{array}\right], \quad B_{z 3}=\left[\begin{array}{ll}
0.1 & 0.2
\end{array}\right], \\
& D_{z 1}=0.1, \quad D_{z 2}=0.1, \quad D_{z 3}=0.1 .
\end{aligned}
$$

With $r_{m}=r_{0}=1, r_{2}=2, r_{M}=r_{3}=3$, and $\alpha_{1}=\alpha_{2}=0.4, \alpha_{3}=0.2$, there is a feasible solution in Theorem 1 with (some matrix solutions for LMI variables are not listed here)

$$
\begin{aligned}
& U_{1}=\left[\begin{array}{cc}
1.4679 & -0.6667 \\
-0.6667 & 2.7987
\end{array}\right], \\
& U_{2}=\left[\begin{array}{cc}
-0.4749 & 1.2218 \\
1.2218 & 1.0524
\end{array}\right], \\
& U_{3}=\left[\begin{array}{cc}
0.2111 & -1.3439 \\
-1.3439 & -7.0066
\end{array}\right] .
\end{aligned}
$$

Hence, we can conclude that the water quality model (22a)-(22b) with (23) is passive with $\gamma=1$ by the switching signal designed by

$$
\sigma= \begin{cases}1, & x(k) \in \bar{\Omega}_{1}, \\ 2, & x(k) \in \bar{\Omega}_{2}, \\ 3, & x(k) \in \Re^{2} \backslash \bar{\Omega}_{1} \backslash \bar{\Omega}_{2},\end{cases}
$$

where

$$
\begin{aligned}
& \bar{\Omega}_{1}=\left\{\left[\begin{array}{ll}
x_{1} & x_{2}
\end{array}\right]^{T} \in \mathfrak{R}^{2}: 1.4679 x_{1}^{2}-1.3334 x_{1} x_{2}+2.7987 x_{2}^{2} \geq 0\right\} \\
& \bar{\Omega}_{2}=\left\{\left[\begin{array}{ll}
x_{1} & x_{2}
\end{array}\right]^{T} \in \Re^{2}:-0.4749 x_{1}^{2}+2.4436 x_{1} x_{2}+1.0524 x_{2}^{2} \geq 0\right\} \backslash \bar{\Omega}_{1} .
\end{aligned}
$$

\section{Conclusions}

In this paper, a simple switching signal design scheme has been investigated to guarantee the passivity property and passive switching control for uncertain discrete switched systems with interval time-varying delay and linear fractional perturbations. A new Lyapunov functional is applied to guaranteed the obtained results. Jensen and Park inequalities combined with delay-partitioning approach are used to improve the conservativeness of the proposed results. The results proposed in this paper are shown to be less conservative than some recent reports from numerical examples. The passivity of a water quality model is guaranteed by selecting a suitable switching signal. 


\section{Author details}

'Department of Marine Engineering, National Kaohsiung Marine University, Kaohsiung, 811, Taiwan, ROC. ${ }^{2}$ Department of Electrical Engineering, National Penghu University of Science and Technology, Penghu, 880, Taiwan, ROC. ${ }^{3}$ Department of Computer Science and Information Engineering, National Quemoy University, Kinmen, 892, Taiwan, ROC. ${ }^{4}$ Department of Applied Informatics and Multimedia, Chia Nan University of Pharmacy and Science, Tainan, 717, Taiwan, ROC.

\section{Acknowledgements}

The research reported here was supported by the Ministry of Science and Technology, ROC under grant no. MOST 104-2221-E-022-003. The authors would like to thank the editor and anonymous reviewers for their helpful comments.

\section{Received: 4 February 2016 Accepted: 30 March 2016 Published online: 11 April 2016}

\section{References}

1. Mahmoud, MS: Switched Time-Delay Systems. Springer, Boston (2010)

2. Sun, Z, Ge, SS: Switched Linear Systems Control and Design. Springer, London (2005)

3. Mahmoud, MS, Nounou, HN, Xia, Y: Robust dissipative control for Internet-based switched systems. J. Franklin Inst. 347, 154-172 (2010)

4. Xiang, W, Xia, J: $H_{\infty}$ finite-time control for switched nonlinear discrete-time systems with norm-bounded disturbance. J. Franklin Inst. 348, 331-352 (2011)

5. Gu, K, Kharitonov, VL, Chen, J: Stability of Time-Delay Systems. Birkhäuser, Boston (2003)

6. Hale, JK, Verduyn Lunel, SM: Introduction to Functional Differential Equations. Springer, New York (1993)

7. Kolmanovskii, VB, Myshkis, A: Applied Theory of Functional Differential Equations. Kluwer Academic, Amsterdam (1992)

8. Du, D: $H_{\infty}$ filter for discrete-time switched systems with time-varying delays. Nonlinear Anal. Hybrid Syst. 4, 782-790 (2010)

9. Hou, L, Zong, $G, W u, Y$ : Observer-based finite-time exponential $l_{2}-l_{\infty}$ control for discrete-time delay systems with uncertainties. Trans. Inst. Meas. Control 35, 310-320 (2013)

10. Lien, $\mathrm{CH}, \mathrm{Yu}, \mathrm{KW}$, Chung, YJ, Chang, $\mathrm{HC}$, Chen, JD, Chung, LY: Exponential stability and robust $H_{\infty}$ control for uncertain discrete switched systems with interval time-varying delay. IMA J. Math. Control Inf. 28, 121-141 (2011)

11. Lien, $C H$, Yu, KW, Chen, JD, Chung, LY: Sufficient conditions for global exponential stability of discrete switched time-delay systems with linear fractional perturbations via switching signal design. Adv. Differ. Equ. 2013, 39 (2013)

12. Mahmoud, MS: Delay-dependent $H_{\infty}$ filtering of a class of switched discrete-time state delay systems. Signal Process. 88, 2709-2719 (2008)

13. Phat, VN, Ratchagit, K: Stability and stabilization of switched linear discrete-time systems with interval time-varying delay. Nonlinear Anal. Hybrid Syst. 5, 605-612 (2011)

14. Sun, YG, Wang, L, Xie, G: Delay-dependent robust stability and $H_{\infty}$ control for uncertain discrete-time switched systems with mode-dependent time delays. Appl. Math. Comput. 187, 1228-1237 (2007)

15. Zhang, L, Shi, P, Basin, M: Robust stability and stabilisation of uncertain switched linear discrete time-delay systems. IET Control Theory Appl. 2, 606-614 (2008)

16. Zhang, WA, Yu, L: Stability analysis for discrete-time switched time-delay systems. Automatica 45, 2265-2271 (2009)

17. Zong, $G, H o u, L, L i$, J: A descriptor system approach to $I_{2}-I_{\infty}$ filtering for uncertain discrete-time switched system with mode-dependent time-varying delays. Int. J. Innov. Comput. Inf. Control 7, 2213-2224 (2011)

18. Lien, $\mathrm{CH}, \mathrm{Yu}, \mathrm{KW}$, Chen, JD, Chung, LY: Switching signal design and passivity analysis for uncertain discrete switched systems with interval time-varying delay. IMA J. Math. Control Inf. 30, 251-263 (2013)

19. Lien, $\mathrm{CH}, \mathrm{Yu}, \mathrm{KW}$, Chen, JD, Chung, LY: Designs of switching signal and passive control for discrete uncertain switched time-delay systems. IMA J. Math. Control Inf. 32, 405-426 (2015)

20. Lien, CH, Yu, KW, Chung, YJ, Chang, HC, Chung, LY, Chen, JD: Switching signal design for global exponential stability of uncertain switched nonlinear systems with time-varying delay. Nonlinear Anal. Hybrid Syst. 5, 10-19 (2011)

21. Pairote, S, Phat, VN: Exponential stability of switched linear systems with time-varying delay. Electron. J. Differ. Equ. $2007,159(2007)$

22. Phat, VN, Botmart, T, Niamsup, P: Switching design for exponential stability of a class of nonlinear hybrid time-delay systems. Nonlinear Anal. Hybrid Syst. 3, 1-10 (2009)

23. Sun, XM, Wang, W, Liu, GP, Zhao, J: Stability analysis for linear switched systems with time-varying delay. IEEE Trans. Syst. Man Cybern. B 38, 528-533 (2008)

24. Chua, LO: Passivity and complexity. IEEE Trans. Circuits Syst. 46, 71-82 (1999)

25. Calcev, G, Gore, R, Neyer, DM: Passivity approach to fuzzy control systems. Automatica 34, 2339-2344 (1998)

26. Mahmoud, MS: Delay-dependent dissipativity of singular time-delay systems. IMA J. Math. Control Inf. 26, 45-58 (2009)

27. Mahmoud, MS, Xie, L: Passivity analysis and synthesis for uncertain time-delay systems. Math. Probl. Eng. 7, 455-484 (2001)

28. Mahmoud, MS, Zribi, M: Passive control synthesis for uncertain systems with multiple-state delays. Comput. Electr. Eng. 28, 195-216 (2002). Special issue on time-delay systems

29. Zhu, J, Zhang, Q, Yuan, Z: Delay-dependent passivity criterion for discrete-time delayed standard neural network model. Neurocomputing 73, 1384-1393 (2010)

30. Zhang, Z, Mou, S, Lam, J, Gao, H: New passivity criteria for neural networks with time-varying delay. Neural Netw. 22 864-868 (2009)

31. Xie, L, Fu, M: Passivity analysis and passification for uncertain signal processing systems. IEEE Trans. Signal Process. 46 2394-2403 (1998)

32. Li, T, Guo, L, Sun, C: Robust stability for neural networks with time-varying delays and linear fractional uncertainties. Neurocomputing 71, 421-427 (2007)

33. Qian, W: Improved stability analysis on delayed neural networks with linear fractional uncertainties. Appl. Math. Comput. 217, 3596-3606 (2010) 
34. Meng, X, Lam, J, Du, B, Gao, H: A delay-partitioning approach to the stability analysis of discrete-time systems. Automatica 46, 610-614 (2010)

35. Park, PG, Ko, JW, Jeong, C: Reciprocally convex approach to stability of systems with time-varying delays. Automatica 47, 235-238 (2011)

36. Kermani, M, Sakly, A: Delay-independent stability criteria under arbitrary switching of a class of switched nonlinear time-delay systems. Adv. Differ. Equ. 2015, 225 (2015)

37. Li, Z, Gao, H, Karimi, HR: Stability analysis and $H_{\infty}$ controller synthesis of discrete-time switched systems with time delay. Syst. Control Lett. 66, 85-93 (2014)

38. Liu, L, Yin, Y, Wang, J, Wu, Q: Stability analysis of discrete-time switched nonlinear systems via T-S fuzzy model approach. Neurocomputing 173, 1967-1971 (2016)

39. Li, J, Zhao, J, Chen, C: Dissipativity and feedback passivation for switched discrete-time nonlinear systems. Syst. Control Lett. 87, 47-55 (2016)

40. Boyd, SP, El Ghaoui, L, Feron, E, Balakrishnan, V: Linear Matrix Inequalities in System and Control Theory. SIAM, Philadelphia (1994)

41. Ibrir, S: Static output feedback and guaranteed cost control of a class of discrete-time nonlinear systems with partial state measurements. Nonlinear Anal. 68, 1784-1792 (2008)

42. Yang, J, Luo, W, Li, G, Zhong, S: Reliable guaranteed cost control for uncertain fuzzy neutral systems. Nonlinear Anal. Hybrid Syst. 4, 644-658 (2010)

43. $\mathrm{Wu}, \mathrm{ZG}, \mathrm{Shi}, \mathrm{P}, \mathrm{Su}, \mathrm{H}, \mathrm{Chu}$, J: Passivity analysis for discrete-time stochastic Markovian jump neural networks with mixed time delays. IEEE Trans. Neural Netw. 22, 1566-1575 (2011)

\section{Submit your manuscript to a SpringerOpen ${ }^{\ominus}$ journal and benefit from:}

- Convenient online submission

- Rigorous peer review

- Immediate publication on acceptance

- Open access: articles freely available online

- High visibility within the field

Retaining the copyright to your article 